\title{
PROJECTIVE LIMITS IN HARMONIC ANALYSIS
}

BY

\author{
WILLIAM A. GREENE
}

\begin{abstract}
A treatment of induced transformations of measures and measurable functions is presented. Given a diagram $\varphi: G \rightarrow H$ in the category of locally compact groups and continuous proper surjective group homomorphisms, functors are produced which on objects are given by $G \rightarrow L^{2}(G), L^{1}(G)$, $M(G), W(G)$, denoting, resp., the $L^{2}$-space, $L^{1}$-algebra, measure algebra, and von Neumann algebra generated by left regular representation of $L^{1}$ on $L^{2}$. All functors but but the second are shown to preserve projective limits; by example, the second is shown not to do so. The category of Hilbert spaces and linear transformations of norm $\leqslant 1$ is shown to have projective limits; some propositions on such limits are given. Also given is a type and factor characterization of projective limits in the category of $W^{*}$-algebras and surjective normal *-algebra homomorphisms.
\end{abstract}

0. Introduction. In the theory of topological groups, two well-known results are the following: Every compact group is the projective limit of closed subgroups of unitary groups $U(n)(n<\infty)$. Every locally compact group contains an open subgroup which is the projective limit of Lie groups. These results indicate why projective limits are of interest-loosely speaking, one has made a reduction to a simpler case. In this paper we investigate whether certain mathematical objects studied by harmonic and functional analysts have projective limit decompositions which reflect decompositions presumed to exist on the group level.

In $\S 1$ we present a treatment of induced transformations of measures and measurable functions. In particular, given $\varphi: G \rightarrow H$ in the category of locally compact groups and continuous, proper (defined within) surjective group homomorphisms, we produce three functors which to $G$ assign, resp., the objects $L^{2}(G), M(G)$, and $L^{1}(G)$, and which to $\Phi$ assign morphisms appropriate to these objects. In $\S 2$ we show that projective (and injective) limits exist in the category of Hilbert spaces and norm-decreasing linear transformations, and present some propositions on such limits. In $\S 3$ a fourth functor is introduced, which to the

Received by the editors March 25, 1974.

AMS (MOS) subject classifications (1970). Primary 43A10, 43A15, 43A20, 22D15, 22D25, 46C10; Secondary 43A95, 46M1 5, 46L10.

Key words and phrases. Functor, category, limit, projective limit, categorical limit preservation, locally compact group, Haar measure, convolution measure algebra, $L^{p}$-space, Banach space, Hilbert space, $C^{*}$-algebra, $W^{*}$-algebra. 
group $G$ assigns $W(G)=$ the von Neumann algebra generated by left regular representation of $L^{1}(G)$ on $L^{2}(G)$. Using techniques from category theory, the functors $G \mapsto L^{2}(G), M(G), W(G)$ are shown to preserve projective limits; by example(s), the functor $G \mapsto L^{1}(G)$ is shown not to do so. Finally, we give a type and factor characterization of projective limits in the category of $W^{*}$-algebras and surjective normal *-algebra homomorphisms.

Throughout we designate the complex numbers by $C$ and the real numbers by $\mathbf{R}$. When describing a function, surjective (resp., injective) is a synonym for onto (resp., one-to-one). Also, we use the terms involutive Banach algebra and Banach *-algebra synonymously, and assume that such an algebra $A$ satisfies: $\|x *\|=\|x\|,\|x y\| \leqslant\|x\|\|y\|, x, y \in A$. A linear map $T$ between Banach spaces is an isometry if $\|T x\|=\|x\|$, all $x$. Finally, an assignment of the objects and morphisms of a category is a functor if the assignment preserves identity morphisms and composition of morphisms.

1. Induced transformations of measures and measurable functions. We seek functors which assign to locally compact groups various function spaces, measure algebras, and operator algebras associated with groups, and which assign to (a suitably restricted class of) group homomorphisms those morphisms appropriate to the codomain categories. In this section we develop some machinery for induced transformations of measures and measurable functions. It turns out that two avenues of development are needed-one for the class of bounded Radon measures $M(G)=C_{0}(G)^{*}$ (and the subclass of $L^{1}$-functions), the second avenue for functions of class $L^{p}, p>1$ (which do not in general determine bounded linear functionals of $C_{0}(G)$ ).

It is our own personal opinion that measure-theoretic technicalities are delicate and vexatious. For instance, a measure defined on the $\sigma$-algebra of Borel sets can have distinct extensions to a larger $\sigma$-algebra-one is immediately confronted with deciding what it will mean to say that two measures are equal. We have not found a treatment of induced transformations of measures and measurable functions which suited our several needs, and which did not slur over the technicalities, so for the sake of completeness we present our own. We have tried to be very precise at critical junctures, elsewhere we are breezier. The Banach spaces $M(G)$ and $L^{p}(G)(p>1)$ are dual spaces to other Banach spaces; we have tried as much as possible to keep our arguments in the framework of linear functionals; this approach is inherently preferable, certainly tidier.

We use Hewitt and Ross [3] as a source; we adopt that work's notation and definitions. For a locally compact Hausdorff space $X, C_{0}(X)$ denotes the continuous $C$-valued functions on $X$ vanishing at infinity, $C_{00}(X)$ denotes 
$\left\{f \in C_{0}(X): \operatorname{supp}(f)\right.$ is compact $\}$. The plus sign + , used as a superscript denotes positive elements. 'Borel set" means an element of the $\sigma$-algebra generated by all open sets. $\xi_{A}$ designates the characteristic function of the subset $A$. If $X$ is a group, $f$ a function on $X, a \in X$, then ${ }_{a} f, f_{a}$ denote the left and right translates of $f,{ }_{a} f(x)=f(a x), f_{a}(x)=f(x a)$. We also adopt the following cautious convention of [3]. To $\Gamma \in C_{0}(X)^{*}$ there is canonically associated a measure $\gamma$ as follows: $\Gamma$ decomposes uniquely $\Gamma=\Gamma_{1}-\Gamma_{2}+i\left(\Gamma_{3}-\Gamma_{4}\right)$ into positive functionals satisfying $\min \left(\Gamma_{1}, \Gamma_{2}\right)=0=\min \left(\Gamma_{3}, \Gamma_{4}\right)$. Let $\gamma_{k}$ be the positive measure constructed from $\Gamma_{k}$ in the Riesz representation theorem, and set $\gamma=\gamma_{1}-$ $\gamma_{2}+i\left(\gamma_{3}-\gamma_{4}\right)$, whose domain in the $\sigma$-algebra $\bigcap_{k=1}^{4} \operatorname{dom}\left(\gamma_{k}\right)$. Then $\Gamma(f)=$ $\int f d \gamma, f \in C_{0}(X)$. We reserve the notation $M(X)$ for the set of measures $\gamma$ so obtained from the elements of $C_{0}(X)^{*}$. Total variation, involution, and convolution are first defined in $C_{0}(X)^{*}$ and thence in $M(X):|\gamma|$ means the measure canonically associated with $|\Gamma|$, etc.

We summarize the Riesz representation theorem as follows. Let a positive linear functional $I$ on $C_{00}(X)$ be given. Define $\bar{I}$ on $M^{+}=$\{lower semicontinuous $g>0\}$ by $\bar{I}(g)=\sup \left\{I(f): 0 \leqslant f \leqslant g, f \in C_{00}(X)\right\}$. For an arbitrary function $h \geqslant 0$, define $\overline{\bar{I}}(h)=\inf \left\{\bar{I}(g): g \geqslant h, g \in M^{+}\right\}$. For $A \subset X$, set $i(A)=\overline{\bar{I}}\left(\xi_{A}\right)$. Then $i$ is a countably additive positive measure on the $\sigma$-algebra $\{S \subset X: i(A)=$ $i(A \cap S)+i(A \cap(X \backslash S))$, all $A \subset X\}$. And $I(f)=\int f d i, f \in C_{00}(X)$ (cf. $\S 11$ of [3]).

Our setting will be: $X$ and $Y$ are locally compact Hausdorff topological spaces and $\varphi: X \rightarrow Y$ is a continuous and proper function. Here by proper we mean that $\varphi^{-1}(C)$ is compact in $X$ if $C$ is compact in $Y$. (Given our setting, it is shown in $\S 10$, Chapitre I of [1] that this definition of proper is equivalent to stipulating that point inverses be compact and $\varphi$ be a closed map. Also it is shown there that $\tilde{\varphi}: X / \operatorname{Ker}(\varphi) \rightarrow \operatorname{Im}(\varphi)$ is a homeomorphism, where $X / \operatorname{Ker}(\varphi)$ denotes the canonical quotient of $X$ by the kernel relation $\operatorname{Ker}(\varphi)=\{(x, y) \in X \times X$ : $\varphi(x)=\varphi(y)\}$.)

Given a positive linear functional $I$ on $C_{00}(X)$, there is induced a positive linear function $\Phi I$ on $C_{00}(Y)$ given by $\Phi I(f)=I(f \circ \varphi), f \in C_{00}(Y)$. Note $\varphi$ being proper implies $f \circ \varphi \in C_{00}(X)$. Let $i$ be the positive Radon measure on $X$ arising from $I$; let $j$ be that on $Y$ arising from $\Phi I$. We compute $j(A)$ for certain $A \subset Y$.

1.1. Theorem. (a) If $A \subset Y$ is $j$-measurable, then $\varphi^{-1}(A)$ is i-measurable.

(b) If $A \subset Y$ is open, or is $j$-measurable of $\sigma$-finite j-measure, then $j(A)=$ $i\left(\varphi^{-1}(A)\right)$.

Proof. We prove (b) first. (1) The case $U \subset Y$ is open: let $D=\{f \in$ 
$\left.C_{00}(Y)^{+}: f \leqslant \xi_{U}\right\}$. Then $\sup \{f: f \in D\}=\xi_{U}$, and $\sup \{f \circ \varphi: f \in D\}=$ $\xi_{\varphi-1}(U)$, where we refer to pointwise suprema. Then

$$
\begin{aligned}
j(U) & =\overline{\bar{\Phi}}\left(\xi_{U}\right)=\overline{\Phi I}\left(\xi_{U}\right) \\
& =\sup \{\Phi I(f): f \in D\}=\sup \{I(f \circ \varphi): f \in D\}=\sup \{\bar{I}(f \circ \varphi): f \in D\} ;
\end{aligned}
$$

by 11.13 of [3], the latter equals $\bar{I}(\sup \{f \circ \varphi: f \in D\})=\bar{I}\left(\xi_{\varphi-1(U)}\right)=i\left(\varphi^{-1}(U)\right)$.

(2) The case $C \subset Y$ is compact: choose open $U \supset C$ such that $j(U \backslash C)$ is finite. Then $j(U)-j(C)=j(U \backslash C)=i\left(\varphi^{-1}(U \backslash C)\right)$ by part (1), and the latter equals $i\left(\varphi^{-1}(U)\right)-i\left(\varphi^{-1}(C)\right)=j(U)-i\left(\varphi^{-1}(C)\right)$. Hence $j(C)=i\left(\varphi^{-1}(C)\right)$.

(3) $A \subset Y$ a Borel set of finite $j$-measure: clearly $\varphi^{-1}(A)$ is Borel. Then

$$
\begin{aligned}
j(A) & =\inf \{j(U): U \text { open, } U \supset A\}=\inf \left\{i\left(\varphi^{-1}(U)\right): U \text { open, } U \supset A\right\} \\
& \geqslant \inf \left\{i(V): V \text { open, } V \supset \varphi^{-1}(A)\right\}=i\left(\varphi^{-1}(A)\right) .
\end{aligned}
$$

Similarly, $j(A)=\sup \{j(C): C$ compact: $C \subset A\} \leqslant i\left(\varphi^{-1}(A)\right)$. So $j(A)=i\left(\varphi^{-1}(A)\right)$.

(4) $j(A)=0$ implies $i\left(\varphi^{-1}(A)\right)=0$ : for any $\epsilon>0$, there is open $U \supset A$ such that $\epsilon>j(U)=i\left(\varphi^{-1}(U)\right) \geqslant i\left(\varphi^{-1}(A)\right)$, showing $\varphi^{-1}(A)$ is $i$-null, and by completeness of $i$, $i$-measurable.

(5) $j$-measurable $A \subset Y$ of finite $j$-measure: we may write $A$ as the disjoint union $A=B \cup N$ of a Borel (in fact, $\sigma$-compact) set $B$ and a $j$-null set $N$. Then $\varphi^{-1}(A)$ is the disjoint union of the Borel set $\varphi^{-1}(B)$ and the $i$-null set $\varphi^{-1}(N)$. Thus $\varphi^{-1}(A)$ is $i$-measurable, and $j(A)=i\left(\varphi^{-1}(A)\right)$.

(6) $j$-measurable $A \subset Y$ of $\sigma$-finite $j$-measure: write $A$ as a countable disjoint union of sets as in (5).

This completes the proof of (b).

(a): In this theory of integration, a set $A$ is measurable $\Leftrightarrow A \cap C$ is measurable for all compact $C$. Given $j$-measurable $A \subset Y$ and compact $C \subset X$, then $A \cap \varphi(C)$ is $j$-measurable of finite $j$-measure $\Rightarrow \varphi^{-1}(A \cap \varphi(C))=\varphi^{-1}(A) \cap$ $\varphi^{-1} \varphi(C)$ is $i$-measurable $\Rightarrow C \cap \varphi^{-1}(A \cap \varphi(C))=C \cap \varphi^{-1}(A)$ is $i$-measurable, and we are done. Q.E.D.

REMARKS. (1) If $N \subset Y$ is locally $j$-null, then $\varphi^{-1}(N)$ is locally $i$-null: given compact $C \subset X$, then $C \cap \varphi^{-1}(N)=C \cap \varphi^{-1}(N \cap \varphi(C))$, and $N \cap \varphi(C)$ is $j$-null, so that $C \cap \varphi^{-1}(N)$ must be $i$-null.

(2) Even assuming $\varphi$ is surjective, we have been unable to show that $i\left(\varphi^{-1}(A)\right)=\infty$ when $j(A)=\infty$. The possible existence of nonnull, locally null sets in $Y$ makes fruitless the attempt to use inner regularity of $j$, and one must return to the definition $i\left(\varphi^{-1}(A)\right)=\overline{\bar{I}}\left(\xi_{\varphi-1}(A)\right)$.

(3) The problem in (2) disappears when $i(X)<\infty$ (that is, $i \in M(X)^{+}$), for then $Y$ open implies $j(Y)=i(X)<\infty$. 
(4) $I$ and $\Phi I$ are either both unbounded or both bounded with the same bound, for $\|\Phi I\|=j(Y)=i(X)=\|I\|$.

1.2. Proposition. If $f \in L^{p}(Y, j), 1 \leqslant p<\infty$, then $f \circ \varphi \in L^{p}(X, i)$, $\int(f \circ \varphi) d i=\int f d j$, and $\|f \circ \varphi\|_{p}=\|f\|_{p}$.

PRoof. We may assume $f \geqslant 0$ everywhere. For $a \in \mathbf{R},\{x: f \circ \varphi(x)>a\}=$ $\varphi^{-1}(\{y: f(y)>a\})$, so $f \circ \varphi$ is $i$-measurable if $f$ is $j$-measurable. Consider the case that $f$ is simple, $f=\Sigma_{k=1}^{n} \alpha_{k} \xi_{A_{k}}$, where $\left\{A_{k}\right\}_{k=1}^{n}$ are pairwise disjoint and of finite $j$-measure. Then $f^{\circ} \varphi=\Sigma \alpha_{k} \xi_{\varphi-1}\left(A_{k}\right)$, and $\int\left(f^{\circ} \varphi\right) d i=\Sigma \alpha_{k} i\left(\varphi^{-1}\left(A_{k}\right)\right)=$ $\Sigma \alpha_{k} j\left(A_{k}\right)=\int f d j$. The general case of measurable $f \geqslant 0$ now follows from the Monotone Convergence Theorem. Finally, $\|f \circ \varphi\|_{p}^{p}=\int\left|f^{\circ} \varphi\right|^{p} d i=\int|f|^{p} \circ d i=$ $\int|f|^{p} d j=\|f\|_{p}^{p}$. Q.E.D.

Let $1<p<\infty$ and let $q$ satisfy $p^{-1}+q^{-1}=1$. Then $L^{p}(X, i)^{*}=$ $L^{q}(X, i)$; we shall use the functional notation $\langle g \mid f\rangle=\int_{X} g(x) f(x) \operatorname{di}(x), g \in L^{p}$, $f \in L^{q}$. This notation is inconsistent with the inner-product defined in the Hilbert space $L^{2}$ (there $\langle g \mid f\rangle=\int_{X} g(x) \overline{f(x)} d i(x)$ ), but our results will not be affected.

1.3. TheOREM. Let $1<p<\infty$. Then there is a surjective linear map $\Psi$ : $L^{p}(X, i) \rightarrow L^{p}(Y, j)$ defined by $\langle\Psi w \mid f\rangle=\langle w \mid f \circ \varphi\rangle$ for $w \in L^{p}(X, i), f \in$ $L^{q}(Y, j)$. Moreover, $\|\Psi\|=1 ; w \geqslant 0$ a.e. implies $\Psi w \geqslant 0$ a.e.; and $\Psi(k \circ \varphi)=$ $k$ for $k \in L^{p}(Y, j)$.

Proof. $\Psi$ is the dual of the Banach space morphism $f \mapsto f \circ \varphi$ of the preceding proposition. Since that morphism is isometric, $\|\Psi\|=1$. Now $\langle\Psi(k \circ \varphi) \mid f\rangle=\langle k \circ \varphi \mid f \circ \varphi\rangle=\int(k \circ \varphi)(f \circ \varphi) d i=\int k f d j=\langle k \mid f\rangle$, showing $\Psi(k \circ \varphi)=k$, hence surjectivity of $\Psi$. In general, $g \in L^{p}$ is $\geqslant 0$ a.e. if and only if $\langle g \mid f\rangle \geqslant 0$ for all $f \geqslant 0$ a.e. in $L^{q}$. Q.E.D.

REMARK. Since $L^{1}$ is in general not the dual of $L^{\infty}$, the preceding cannot apply to the case $p=1$.

Let $C_{0}(X)$ be given the uniform norm. The map $\varphi: X \rightarrow Y$ induces a linear map $C_{0}(Y) \rightarrow C_{0}(X)$ given by $f \mapsto f \circ \varphi$, in turn inducing a linear map $\Phi: C_{0}(X)^{*} \rightarrow C_{0}(Y)^{*}$ given by $\Phi \Gamma(f)=\Gamma\left(f^{\circ} \varphi\right)$. Clearly $\|\Phi\| \leqslant 1$, and it follows from Remark 4 after Theorem 1.1 that, in fact, $\|\Phi\|=1$, since $\Phi$ is isometric on the positive measures in $C_{0}(X)^{*}$.

1.4. Let $\Gamma \in C_{0}(X)^{*}$, let $\Gamma=\Gamma_{1}-\Gamma_{2}+i\left(\Gamma_{3}-\Gamma_{4}\right)$ be its canonical decomposition into positive functionals satisfying $\min \left(\Gamma_{1}, \Gamma_{2}\right)=0=\min \left(\Gamma_{3}, \Gamma_{4}\right)$, let $\gamma=\gamma_{1}-\gamma_{2}+i\left(\gamma_{3}-\gamma_{4}\right)$ be the measures canonically constructed from $\Gamma$, $\gamma_{n}$ being constructed from $\Gamma_{n}, n=1,2,3,4$, as in the Riesz representation theorem. The map $\Phi$ does not in general preserve the lattice structure of 
$\left\{\Gamma \in C_{0}(X)^{*}: f\right.$ is real-valued $\left.\Rightarrow \Gamma(f) \in \mathbf{R}\right\}$ (see 1.6 below). Consequently, the canonical decomposition of $\Phi \Gamma$ into minimal positive parts is not in general given by $\Phi \Gamma_{1}-\Phi \Gamma_{2}+i\left(\Phi \Gamma_{3}-\Phi \Gamma_{4}\right)$, although the two agree as functionals on $C_{0}(Y)$. It follows that if $\Phi \gamma$ denotes the measure canonically constructed from $\Phi \Gamma$, and $\Phi \gamma_{n}$ denotes that constructed from $\Phi \Gamma_{n}$ (and calculated in Theorem 1.1), then the measure $\Phi \gamma$ is not in general equal to the measure $\Phi \gamma_{1}-\Phi \gamma_{2}+i\left(\Phi \gamma_{3}-\Phi \gamma_{4}\right)$, as their domains may be different.

These two measures do agree on their common domain, however, and in particular, for Borel $A \subset Y$, we have $(\Phi \gamma)(A)=\gamma\left(\varphi^{-1}(A)\right)$, by Theorem 1.1. Also, if $k$ is a bounded Borel measurable function on $Y$, then $k$ is in the $L^{1}$-class of $|\Phi \gamma|$ and of $\Phi \gamma_{n}, n=1,2,3,4$, and we can conclude

$$
\begin{aligned}
\int k d \Phi \gamma & =\int k d \Phi \gamma_{1}-\int k d \Phi \gamma_{2}+i \int k d \Phi \gamma_{3}-\int k d \Phi \gamma_{4} \\
& =\int k \circ \dot{\varphi} d \gamma_{1}-\int k^{\circ} \varphi d \gamma_{2}+i \int k^{\circ} \varphi d \gamma_{3}-i \int k \circ \varphi d \gamma_{4} \\
& =\int k \circ \varphi d \gamma .
\end{aligned}
$$

Caveat. One must be wary of expressions $\int k d \Phi \gamma=\int k^{\circ} \varphi d \gamma$ for unbounded Borel functions $k$ on $Y$. For suppose $\Phi \gamma_{1}=\Phi \gamma_{2}=\Phi \gamma_{3}=\Phi \gamma_{4}$; it follows that $\Phi \gamma$ is the zero Borel measure. We can find positive unbounded Borel $k$ on $Y$ such that $\int k d \Phi \gamma_{n}=\infty(n=1,2,3,4)$. Then the first term in (*) becomes $\int k d 0=0$, whereas the second term does not make sense.

Having highlighted the hazards of computation, we shall begin to slur over matters somewhat ourselves and write $\Phi: M(X) \rightarrow M(Y)$.

The total variation $|\Gamma|$ of an element $\Gamma \in C_{0}(X)^{*}$ is defined first for positive functions $f \in C_{0}(X)^{+}$by $|\Gamma|(f)=\sup \left\{|\Gamma(g)|: g \in C_{0}(X),|g| \leqslant f\right\}$ and then extended to $C_{0}(X)$ by linearity. The first assertion of the following lemma follows from this definition; the equivalence cited is pointed out in 14.8 of [3].

Lemma. Let $\Gamma \in C_{0}(X)^{*}$ and let $\gamma$ be its associated measure. Then $|\Phi \Gamma|(f) \leqslant$ $(\Phi|\Gamma|)(f)$, all $f \in C_{0}(Y)^{+}$. Equivalently, $|\Phi \gamma|(C) \leqslant \Phi|\gamma|(C)$, all compact $C \subset Y$.

DEFinition. Given $I$ and $i$ as before, and given $\gamma \in M(X)$, then $\gamma$ is absolutely continuous with respect to $i$, written $\gamma \ll i$, provided $i(F)=0$ implies $|\gamma|(F)=0$ for compact $F \subset X$.

1.5. THEOREM. Let $I, i$, and $j$ be as in 1.1. The map $\Phi: M(X) \rightarrow M(Y)$ sends $L^{1}(X, i)$ surjectively onto $L^{1}(Y, j)$.

Proof. We first show $\gamma \ll i$ implies $\Phi \gamma \ll j$. Let $F \subset Y$ be compact and let $j(F)=0$. Then $i\left(\varphi^{-1}(F)\right)=0$, so that $0=|\gamma|\left(\varphi^{-1}(F)\right)=\Phi|\gamma|(F)$; the 
preceding lemma gives $|\Phi \gamma|(F)=0$, so $\Phi \gamma \ll j$. Under the identification of $L^{1}(X, i)$ with $\{\gamma \in M(X): \gamma \ll i\}$, $\Phi$ sends $L^{1}(X, i)$ into $L^{1}(Y, j)$. Thus if $f \in$ $L^{1}(X, i)$, we may write $\Phi(f)$ for that element of $L^{1}(Y, j)$ satisfying $\int k \Phi(f) d j=$ $\int(k \circ \varphi) f d i$, all $k \in C_{0}(Y)$. Now let $g \in L^{1}(Y, j)$, so that $g \circ \varphi \in L^{1}(X, i)$; if $k$ $\in C_{0}(Y)$, then $\int k \Phi(g \circ \varphi) d j=\int(k \circ \varphi)(g \circ \varphi) d i=\int(k g) \circ \varphi d i=\int k g d j$, showing $\Phi(g \circ \varphi)=g$ and, hence, the surjectivity of $\left.\Phi\right|_{L^{1}(X, i)}$. Q.E.D.

1.6. EXAMPLE. Let $\varphi: T^{1} \rightarrow\{*\}$ be the constant function from the circle to a singleton set.

(a) Let $L, M \in C_{0}\left(T^{1}\right)^{*}$ be nonzero, positive, and satisfying $\min (L, M)=0$. Since $\Phi$ is isometric on positive functionals, $\Phi L$ and $\Phi M$ can be identified with nonzero positive real numbers, hence $\min (\Phi L, \Phi M) \neq 0$.

(b) The statement $\Phi|\gamma|=|\Phi \gamma|$ is not valid for arbitrary $\gamma \in M(X)$. If it were, $\Phi$ would be isometric: $\|\Phi \gamma\|=|\Phi \gamma|(Y)=\Phi|\gamma|(Y)=|\gamma|(X)=\|\gamma\|$. In our example $\Phi$ cannot be isometric.

Proposition. $\Phi$ and $\Psi$ agree on $L^{1}(X, i) \cap L^{p}(X, i), 1<p<\infty$.

Proof. Let $w \in L^{1}(X) \cap L^{p}(X)$ and, as before, let $\Phi(w)$ denote that element of $L^{1}(Y, j)$ satisfying $\int k \Phi(w) d j=\int(k \circ \varphi) w d i$, all $k \in C_{0}(Y)$. If $k \in$ $C_{00}(Y)$, so that $k \in C_{0}(Y) \cap L^{q}(Y, j)$, then $\int k \Phi(w) d j=\int(k \circ \varphi) w d i=\langle k \circ \varphi$ $|w\rangle=\langle k \mid \Psi w\rangle$, considering $k \circ \varphi \in L^{q}(X, i)$. Thus $\left|\int k \Phi(w) d j\right| \leqslant\|k\|_{q}\|\Psi w\|_{p}$, and we conclude that $\Phi w \in L^{q}(Y, j)^{*}=L^{p}(Y, j)$ and also that $\Phi w=\Psi w$. Q.E.D.

We observe that $L^{1}(X) \cap L^{p}(X)$ is a Banach space under $\|f\|_{1, p}=\|f\|_{1}+$ $\|f\|_{p}$, so the restriction of $\Phi$ (or $\Psi$ ) to this space is a linear map of norm $\leqslant 1$ into the Banach space $L^{1}(Y) \cap L^{p}(Y)$.

Now let $G$ and $H$ be locally compact (Hausdorff) groups and let $\varphi: G \rightarrow H$ be a continuous proper surjective group homomorphism. Let $\Lambda_{G}, \Lambda_{H}$ be left Haar integrals on $G$ and $H$, respectively. Then $\Phi \Lambda_{G}$ is left translation invariant on $C_{00}(H)$ and can be identified with $\Lambda_{H}$ : if $f \in C_{00}(H)$ and $b \in H$, by surjectivity of $\varphi$, choose $a \in G$ such that $\varphi(a)=b$. Then $\left({ }_{b} f\right) \circ \varphi={ }_{a}(f \circ \varphi)$, so $\dot{\Phi} \Lambda_{G}\left({ }_{b} f\right)=$ $\Lambda_{G}\left(\left(_{b} f\right) \circ \varphi\right)=\Lambda_{G}(f \circ \varphi)=\Phi \Lambda_{G}(f)$. Moreover, if $G$ is compact and $\lambda_{G}$ is normalized Haar measure on $G$, then $\lambda_{H}=\Phi \lambda_{G}$ is normalized, for $\Phi \lambda_{G}(H)=$ $\dot{\lambda}_{G}\left(\varphi^{-1}(H)\right)=\lambda_{G}(G)=1$.

Let $L, M \in C_{0}(G)^{*}$. Involution $L^{\sim}$ and convolution $L * M$ are defined as follows: for $f \in C_{0}(G)$, set $f^{\sim}(x)=\overline{f\left(x^{-1}\right)}$ and define $L^{\sim}(f)=\overline{L\left(f^{\sim}\right)}$. Define $\bar{M}: C_{0}(G) \rightarrow C_{0}(G)$ by $\left.\bar{M}(f)(x)=M{ }_{x} f\right)$, define $L * M=L \circ \bar{M}$. These definitions of involution and convolution give rise to the usual ones for measures and $L^{1}$-functions. It is straightforward to verify that the transformation $\Phi$ preserves involution and convolution.

We summarize our results in the following theorem; only the third part 
has not already been shown, and it is readily verified.

1.7. THEOREM. Let $\varphi: G \rightarrow H$ be a continuous proper surjective group homomorphism between locally compact groups.

(1) There exists a *algebra homomorphism $\Phi: M(G) \rightarrow M(H)$ of norm 1 sending $L^{1}\left(G, \lambda_{G}\right)$ onto $L^{1}\left(H, \lambda_{H}\right)$, defined by

$$
\int_{H} f d \Phi \mu=\int_{G} f^{\circ} \varphi d \mu, \quad f \in C_{0}(H) .
$$

(2) For $1<p<\infty$, there exists a surjective linear map $\Psi: L^{p}\left(G, \lambda_{G}\right) \rightarrow$ $L^{p}\left(H, \lambda_{H}\right)$ of norm 1 defined by

$$
\langle\Psi w \mid f\rangle=\left\langle w \mid f^{\circ} \varphi\right\rangle, \quad w \in L^{p}(G), \quad f \in L^{q}(H), \quad p^{-1}+q^{-1}=1 .
$$

Moreover, $\Psi$ agrees with $\Phi$ on their common domain.

(3) Restricting our attention to those group morphisms indicated, the assignments in (1) and (2) are functorial.

$L^{2}(G)$ is a left module over $M(G)$ under $(\mu * f)(x)=\int f\left(y^{-1} x\right) d \mu(y) ;$ this action satisfies $\|\mu * f\|_{2} \leqslant\|\mu\|\|f\|_{2}$ (and analogously for all $1 \leqslant p \leqslant \infty$ ). In our setting of a proper surjective morphism $\varphi: G \rightarrow H$ between locally compact groups, we wish to show $\Phi \mu * \Psi f=\Psi(\mu * f)$. We will need to apply the Fubini theorem.

Let $f, g \in L^{2}(G)$ be $\geqslant 0$ and be Borel measurable, let $\mu \in M(G)$, and define $\alpha: G \times G \rightarrow C$ by $\alpha(x, y)=f\left(x^{-1} y\right) g(y)$. Then $\alpha$ is a Borel measurable (approximate $f$ and $g$ by positive simple Borel measurable functions; a pointwise limit of Borel functions is again Borel), and also $\alpha$ has $\sigma$-finite support with respect to $|\mu| \times \lambda_{G}(\alpha$ is supported in $G \times(\operatorname{supp} g))$. Consequently, we can apply the Fubini theorem to conclude

$$
\int_{G} \int_{G} f\left(x^{-1} y\right) g(y) d \mu(x) d \lambda_{G}(y)=\int_{G} \int_{G} f\left(x^{-1} y\right) g(y) d \lambda_{G}(y) d \mu(x)
$$

provided, say $\int_{G} \int_{G} f\left(x^{-1} y\right) g(y) d|\mu|(x) d \lambda_{G}(y)$ is finite (note that in the last integral it is the total variation $|\mu|$ that appears). (Cf. 13.10, 14.25 of [3].)

1.8. Proposition. Let $\mu \in M(G), f \in L^{2}(G)$; Then $\Phi \mu * \Psi f=\Psi(\mu * f)$.

PRoof. We show $\langle\Phi \mu * \Psi f \mid k\rangle=\langle\Psi(\mu * f) \mid k\rangle$, all $k \in L^{2}(H)$. We may assume $f$ and $k$ are positive and Borel measurable. Then

$$
\begin{gathered}
\int_{H} \int_{H}(\Psi f)\left(h^{-1} x\right) k(x) d|\Phi \mu|(h) d \lambda_{H}(x)=\int_{H} \int_{H}(\Psi f)\left(h^{-1} x\right) d|\Phi \mu|(h) k(x) d \lambda_{H}(x) \\
=\int_{H}(|\Phi \mu| * \Psi f)(x) k(x) d \lambda_{H}(x)=\langle|\Phi \mu| * \Psi f \mid k\rangle
\end{gathered}
$$

is finite, hence 


$$
\begin{aligned}
\langle\Phi \mu * \Psi f \mid k\rangle & =\int_{H} \int_{H}(\Psi f)\left(h^{-1} x\right) k(x) d \Phi \mu(h) d \lambda_{H}(x) \\
& =\int_{H} \int_{H}(\Psi f)\left(h^{-1} x\right) k(x) d \lambda_{H}(x) d \Phi \mu(h) \\
& =\int_{H}\left\langle_{h^{-1}}(\Psi f) \mid k\right\rangle d \Phi \mu(h)=\int_{H}\left\langle\left.\Psi f\right|_{h} k\right\rangle d \Phi \mu(h) .
\end{aligned}
$$

Since the function $H \rightarrow L^{2}(H)$ given by $h \mapsto{ }_{h} k$ is bounded and continuous, the integrand appearing in the last integral is a continuous bounded $C$-valued function on $H$, and we conclude as in 1.4 that the last integral equals

$$
\begin{aligned}
& \left.\int_{G}\left\langle\left.\Psi f\right|_{\varphi g} k\right\rangle d \mu(g)=\int_{G}\left\langle f||_{\varphi g} k\right) \circ \varphi\right\rangle d \mu(g)=\int_{G}\left\langle\left. f\right|_{g}(k \circ \varphi)\right\rangle d \mu(g) \\
& \quad=\int_{G}\left\langle_{g^{-1}} f \mid k \circ \varphi\right\rangle d \mu(g)=\int_{G} \int_{G} f\left(g^{-1} y\right)(k \circ \varphi)(y) d \lambda_{G}(y) d \mu(g) .
\end{aligned}
$$

We justify application of the Fubini theorem as before; , the last integral equals

$$
\begin{aligned}
\int_{G} \int_{G} f\left(g^{-1} y\right) d \mu(g)(k \circ \varphi)(y) d \lambda_{G}(y) & =\int_{G}(\mu * f)(y)(k \circ \varphi)(y) d \lambda_{G}(y) \\
& =\langle\mu * f \mid k \circ \varphi\rangle=\langle\Psi(\mu * f) \mid k\rangle \text {. Q.E.D. }
\end{aligned}
$$

REMARKS. (1) Defining the modular function by $\int f_{a-1}=\Delta(a) \int f$ for $f \in$ $C_{00}$, the identity $\Delta_{H}{ }^{\circ} \varphi=\Delta_{G}$ is obtained.

(2) $\Phi$ and $\Psi$ "preserve" translation in the sense that, for example, $\Psi\left(w_{a}\right)=$ $(\Psi w)_{\varphi a}$, for $w \in L^{p}(G), a \in G$.

(3) If $G$ is compact, then the group operation map $\alpha: G \times G \rightarrow G, \alpha(x, y)$ $=x y$, is a proper continuous surjection. If $\mu, \rho \in M(G)$, then the product measure $\mu \times \rho$ belongs to $M(G \times G)$. The measure on $G$ induced from $\mu \times \rho$ via $\alpha$ is the convolution $\mu * \rho$. In particular, Haar measure $\lambda_{G \times G}=\lambda_{G} \times \lambda_{G}$ on $G \times G$ induces Haar measure $\lambda_{G}$ on $G$ via $\alpha$.

2. Projective limits and Hilbert spaces. In this section we obtain a slightly surprising result on the existence of projective limits of Hilbert spaces, and present some propositions about such projective limits. We shall assume that the reader is familiar with the categorical concepts of product, equalizer, limit, etc. (see [4]).

DEFinition. A linear contraction is a linear transformation of norm $\leqslant 1$ between Banach spaces.

In the class of all Banach spaces, Hilbert spaces are those whose geometry duplicates that of ordinary Euclidean space, and in that internal sense they are the most tractable of Banach spaces. The external (that is, categorical) behavior of Hilbert spaces is a disappointment: 
2.1. Proposition. In the category $H=$ (Hilbert spaces, linear contractions), no Hilbert spaces serve as the product or coproduct of two copies of $C$.

PRoof. Let $H_{1}=H_{2}=C$ with its usual Hilbert space structure, $\langle x \mid y\rangle=$ $x \bar{y}, x, y \in C$. Suppose $H$ serves as their categorical product, with projections $f_{j}: H \rightarrow H_{j}, j=1,2$. Then $f_{j}$ is a linear functional of norm $\leqslant 1$, hence $f_{j}(x)=$ $\left\langle x \mid e_{j}\right\rangle, x \in H$, where $\left\|e_{j}\right\| \leqslant 1, e_{j} \in H$. Let $A=C$, define $\alpha_{j}: A \rightarrow H_{j}$ by

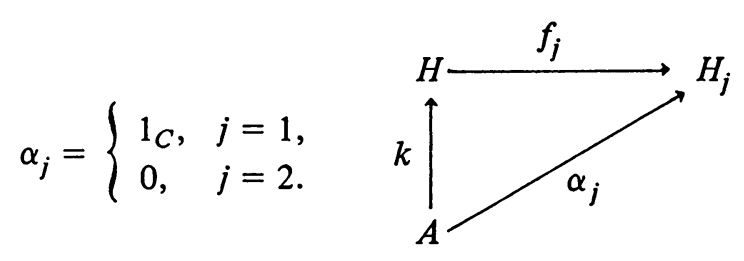

Let $k: A \rightarrow H$ be the unique fill-in determined by $\alpha_{1}, \alpha_{2}$. Then $1=\alpha_{1}(1)=$ $f_{1}(k(1))=\left\langle k(1) \mid e_{1}\right\rangle$ implies $k(1)=e_{1}$, and is a unit vector, since both have norm $\leqslant 1$. Similarly $e_{2}$ is a unit vector. $0=\alpha_{2}(1)=f_{2}(k(1))=\left\langle k(1) \mid e_{2}\right\rangle=\left\langle e_{1} \mid e_{2}\right\rangle$ implies $e_{1} \perp e_{2}$. Now let $B=C$, define $\beta_{j}: B \rightarrow H_{j}$ to be $1_{C}$ for $j=1,2$; let $\tau: B \rightarrow H$ be the fill-in determined by the $\beta$ 's. Then $\tau$ a contraction implies $\|\tau(1)\| \leqslant 1$, whereas $1=\beta_{j}(1)=f_{j}(\tau(1))=\left\langle\tau(1) \mid e_{j}\right\rangle$ for $j=1,2$ implies $\|\tau(1)\|$ $\geqslant 2^{1 / 2}$, a contradiction. The claim for coproducts now follows by taking dual objects and dual morphisms (of Banach spaces), for a Hilbert space may be identified with its topological dual, and the dual of a linear contraction is a linear contraction. Q.E.D.

An argument with a similar flavor shows that in the larger category (Hilbert spaces, continuous linear transformations), no Hilbert space will serve as the product or coproduct of countably many copies of $C$ (although finite products andcoproducts do exist-namely, what is customarily called Hilbert space direct sum).

It is known that the category $B=$ (Banach spaces, linear contractions) has products, coproducts, equalizers, and coequalizers, so that $B$ is complete and cocomplete, that is, any (set-indexed) diagram in $B$ has a limit and a colimit in $B$.

2.2. In particular, projective limits exist in $B$ (and at this point we amplify): assume that $D$ is an upward directed set; that for $\delta \in D, A_{\delta}$ is a $B$-object; that for $\alpha \leqslant \beta$ in $D, A_{\alpha} \stackrel{T_{\alpha \beta}}{\stackrel{1}{~}} A_{\beta}$ is a $B$-morphism; that for $\alpha \leqslant \beta \leqslant \gamma$ in $D, T_{\alpha \beta} T_{\beta \gamma}$ $=T_{\alpha \gamma}$. Such a diagram of objects and morphisms is called a projective system in $B$. Define the set $A$ and the functions $\lambda_{\alpha}: A \rightarrow A_{\alpha}$ by

$$
A=\left\{\left(a_{\delta}\right) \in \times A_{\delta}: \sup _{D}\left\|\alpha_{\delta}\right\|=\left\|\left(a_{\delta}\right)\right\|<\infty, \alpha \leqslant \beta \Rightarrow T_{\alpha \beta} \alpha_{\beta}=a_{\alpha}\right\},
$$

$$
\lambda_{\alpha}\left(\left(a_{\delta}\right)\right)=a_{\alpha} .
$$


One verifies that $A$, equipped with coordinate operations and the norm indicated, is a $B$-object, and that the $\lambda_{\alpha}$.s are $B$-morphisms satisfying $T_{\alpha \beta} \circ \lambda_{\beta}=\lambda_{\alpha}$. Moreover, if $B$ is a $B$-object, if for $\delta \in D, B \stackrel{f_{\delta}}{\longrightarrow} A_{\delta}$ is a $B$-morphism, and if $\left\{f_{\delta}\right\}$ satisfy $T_{\alpha \beta} \circ f_{\beta}=f_{\alpha}$ when $a \leqslant \beta$, then there exists a unique $B$-morphism $\kappa: B \rightarrow A$ satisfying $\lambda_{\delta} \circ \kappa=f_{\delta}$, all $\delta$; the map $\kappa$ is given by $\kappa(b)=\left(f_{\delta}(b)\right)$.

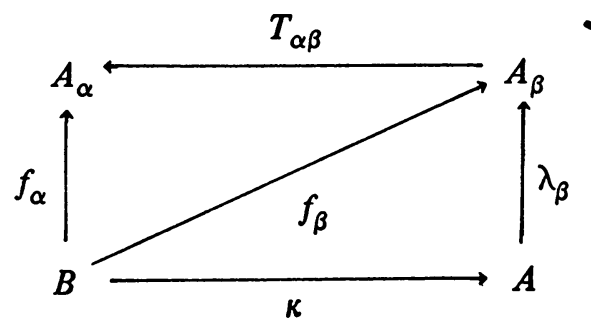

In categorical parlance, any object $A$ with morphisms $\lambda_{\alpha}$ satisfying the properties just enumerated is called the projective limit of the given projective system (any two must be isomorphic), although we shall use the concrete version shown and write $A=$ pro-lim $A_{\delta}$. The definition of an injective system is obtained by reversing all arrows in a projective system. Its colimit is called an injective limit, and it satisfies the universal property dual to the one given above; the injective limit can be realizéd as a quotient of the coproduct of the $A_{\delta}$ 's.

We note that given a projective system in either of the subcategories $B^{\prime}=$ (Banach algebras, contractive algebra homomorphisms) or $B^{\prime}=$ (Banach *-algebras, contractive *-algebra homomorphisms), the set $A$ defined above can be given the structure of a $B^{\prime}$-object, $\lambda_{\alpha}$ becomes a $B^{\prime}$-morphism, hence $\left\{A \stackrel{\lambda_{\alpha}}{\longrightarrow} A_{\alpha}\right\}$ is the projective limit in $B^{\prime}$ of the system $\left\{A_{\alpha} \stackrel{T_{\alpha \beta}}{\longleftarrow} A_{\beta}\right\}$.

The fact that no products or coproducts of $C$ exist in $H=$ (Hilbert spaces, linear contractions) augurs that we should not expect this category to have many limits. It is something of a surprise, then, to discover that

2.3. THEOREM. The category $H=$ (Hilbert spaces, linear contractions) has projective limits. In fact, $B \supset \mathrm{H}$, and the limit, as calculated in $B$, of a projective system in $\mathrm{H}$ already belongs to $\mathrm{H}$.

Proof. Let $\left\{H_{\alpha} \stackrel{T_{\alpha \beta}}{\longleftarrow} H_{\beta}: \alpha \leqslant \beta\right.$ in $\left.D\right\}, D$ directed, be a projective system in $H$, and let $H=$ pro-lim $H_{\delta}=$ the $B$-computed projective limit. Let $\bar{x}=$ $\left(x_{\delta}\right) \in$ pro-lim $H_{\delta}$; since $T_{\alpha \beta}$ is contractive, $\left\|x_{\alpha}\right\|=\left\|T_{\alpha \beta} x_{\beta}\right\| \leqslant\left\|x_{\beta}\right\|$ when $\alpha \leqslant$ $\beta$, whence $\|\bar{x}\|=\sup _{D}\left\|x_{\delta}\right\|=\lim _{\delta \uparrow}\left\|x_{\delta}\right\|$. A Banach space is a Hilbert space if and only if its norm satisfies the parallelogram condition:

$$
\|a+b\|^{2}+\|a-b\|^{2}=2\left(\|a\|^{2}+\|b\|^{2}\right) .
$$


We conclude that $H$ is a Hilbert space by considering limits $\|\bar{x}\|=\lim _{\delta \uparrow}\left\|x_{\delta}\right\|$. As every $B$-morphism between $H$-objects is already an $H$-morphism (that is, $H$ is full in $B$ ), we are done. Q.E.D.

If the norm in a Banach space satisfies the parallelogram condition, then an inner-product is defined by the polarization formula $4\langle x \mid y\rangle=\Sigma_{n=1}^{4} i^{n}\left\|x+i^{n} y\right\|^{2}$; it follows that the inner-product in pro-lim $H_{\delta}$ is given by $\left\langle\left(x_{\delta}\right) \mid\left(y_{\delta}\right)\right\rangle=$ $\lim _{\delta \uparrow}\left\langle x_{\delta} \mid y_{\delta}\right\rangle$.

By taking dual objects and dual morphisms (of Banach spaces), on identifying a Hilbert space with its topological dual, and applying the theorem, we obtain

COROLlary. The category $H$ has injective limits. The colimit (as calculated in H) of an injective system in $H$ serves as the colimit in the larger category (reflexive Banach spaces, linear contractions).

We now collect some results on projective limits in $H$ which are of independent interest, but which will not be needed in $\S 3$.

2.4. Proposition: Let $\left\{H_{\alpha} \stackrel{T_{\alpha \beta}}{\longleftarrow} H_{\beta}: \alpha, \beta \in D, D\right.$ directed $\}$ be a projective system in $H$ with limit $H \stackrel{\lambda_{\delta}}{\longrightarrow} H_{\delta}$. If each $T_{\alpha \beta}$ is an isometry, then each $\lambda_{\delta}$ is an isometry.

Proof. Let $\bar{x}=\left(x_{\delta}\right)$ be an element of $H$. Let $\alpha, \beta$ be any two elements of $D$ and choose $\gamma \in D$ such that $\gamma \geqslant \alpha, \beta$. Then $\left\|x_{\alpha}\right\|=\left\|T_{\alpha \gamma} x_{\gamma}\right\|=\left\|x_{\gamma}\right\|=$ $\left\|T_{\beta \gamma} x_{\gamma}\right\|=\left\|x_{\beta}\right\|$. Thus each coordinate of $\bar{x}$ has the same norm and $\|\bar{x}\|$ is this common value. Then $\lambda_{\delta}(\bar{x})=x_{\delta}$ is an isometric map. Q.E.D.

Definition. A co-isometry is a morphism $T: X \rightarrow Y$ of Hilbert spaces whose Hilbert space adjoint $T^{*}: Y \rightarrow X$ is an isometry. It follows that $T: X \rightarrow$ $Y$ is a co-isometry $\Leftrightarrow T T^{*}=1_{Y}$.

If $Y \stackrel{S}{\longrightarrow} X \stackrel{T}{\longrightarrow} Y$ is in $H$ and $T S=1_{Y}$, then $S$ is isometric, $T$ is co-isometric. Reason: if for some $y \in Y,\|S y\|<\|y\|$, then $T S y=y$ implies $\|T\|>1$, a contradiction to $T$ being contractive. Thus $\|S y\|=\|y\|$, all $y ; S$ is isometric. And $S^{*} T^{*}=1_{Y}^{*}=1_{Y} \Rightarrow T^{*}$ isometric $\Rightarrow T$ co-isometric. (In oral communication, J. Deddens showed us that $S$ and $T$ must be Hilbert space adjoints of each other; he argued by splitting apart the unitary and "pure isometry" parts of $S$ and by considering operator matrices. In the next proposition we prove a special case of this result, in the context of projective limits.) We need the following two facts, both generally known to operator theorists:

(1) Let $T: X \rightarrow Y$ be co-isometric, let $T x=y$. If $\|x\|=\|y\|$, then $x=$ $T^{*} y$.

(2) If $T$ is co-isometric, then $\{x \in X:\|x\|=\|T x\|\}=\operatorname{ran} T^{*}=(\text { null } T)^{\perp}$. 
2.5. Proposition. Let $\left\{H_{\alpha} \stackrel{T_{\alpha \beta}}{\longleftarrow} H_{\beta}: \alpha, \beta \in D, D\right.$ directed $\}$ be a projective system in $H$ with limit $H \stackrel{\lambda_{\delta}}{\longrightarrow} H_{\delta}$. If each $T_{\alpha \beta}$ is a co-isometry, then each $\lambda_{\delta}$ is a co-isometry.

Proof. Fix $\beta \in D$. Define $k_{\beta}=1_{H_{\beta}}$. For other $\delta \in D$, choose $\gamma \geqslant \beta, \delta$ and define $k_{\delta}: H_{\beta} \rightarrow H_{\delta}$ by $k_{\delta}=T_{\delta \gamma} T_{\beta \gamma}^{*}$. Then $k_{\delta}$ is well defined: if also $\eta \geqslant \beta, \delta$, choose $\xi \geqslant \gamma, \eta$. Then

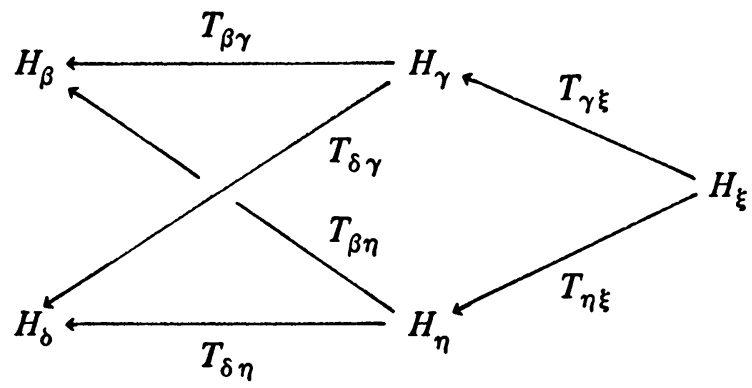

$$
\begin{aligned}
T_{\delta \gamma} T_{\beta \gamma}^{*} & =T_{\delta \gamma} 1_{H_{\gamma}} T_{\beta \gamma}^{*}=T_{\delta \gamma} T_{\gamma \xi} T_{\gamma \xi}^{*} T_{\beta \gamma}^{*}=T_{\delta \gamma} T_{\gamma \xi}\left(T_{\beta \gamma} T_{\gamma \xi}\right)^{*} \\
& =T_{\delta \xi} T_{\beta \xi}^{*}=T_{\delta \eta} T_{\eta \xi}\left(T_{\beta \eta} T_{\eta \xi}\right)^{*}=T_{\delta \eta} T_{\beta \eta}^{*},
\end{aligned}
$$

showing $k_{\delta}$ does not depend on $\gamma \geqslant \beta, \delta$. And $\mu \leqslant \delta$ implies $T_{\mu \delta} k_{\delta}=T_{\mu \delta} T_{\delta \gamma} T_{\beta \gamma}^{*}=$ $k_{\mu}$. Thus $\left\{H_{\beta} \stackrel{k_{\delta}}{\longrightarrow} H_{\delta}: \delta \in D\right\}$ commutes with the system; by universality, there is $\kappa_{\beta}: H_{\beta} \rightarrow H$ such that $\lambda_{\delta} \kappa_{\beta}=k_{\delta}$, all $\delta$; in particular, $\lambda_{\beta} \kappa_{\beta}=1_{H_{\beta}}, \lambda_{\beta}$ is co-isometric, $\kappa_{\beta}$ is isometric.

We will also show that $\lambda_{\beta}$ and $\kappa_{\beta}$ are adjoints. Let $y \in H_{\beta}$, denote $\lambda_{\beta}^{*}(y)=\left(w_{\delta}\right)$ $\in H=$ pro-lim $H_{\delta}$; we identify the coordinates $w_{\delta}$. Now $w_{\beta}=\lambda_{\beta}\left(\left(w_{\delta}\right)\right)=$ $\lambda_{\beta} \lambda_{\beta}^{*}(y)=y$. And $\lambda_{\beta}^{*}$ isometric implies $\|y\|=\left\|\left(w_{\delta}\right)\right\|=\sup \left\|w_{\delta}\right\|$; since this is an increasing net of positive real numbers, we conclude $\|y\|=\left\|w_{\beta}\right\|=\left\|w_{\gamma}\right\|$, all $\gamma \geqslant \beta$. But $T_{\beta \gamma} w_{\gamma}=w_{\beta}$, and the first fact cited above shows $w_{\gamma}=T_{\beta \gamma}^{*} w_{\beta}$, all $\gamma \geqslant \beta$. For any $\delta \in D$, choose $\gamma \geqslant \beta$, $\delta$, then $w_{\delta}=T_{\delta \gamma} w_{\gamma}=T_{\delta \gamma} T_{\beta \gamma}^{*} w_{\beta}=k_{\delta} w_{\beta}$ $=k_{\delta}(y)$. Then $\lambda_{\beta}^{*}(y)=\left(w_{\delta}\right)=\left(k_{\delta}(y)\right)=\kappa_{\beta}(y)$, that is $\lambda_{\beta}^{*}=\kappa_{\beta}$. Q.E.D.

REMARK. We summarize the first part of the proof. Each $T_{\alpha \beta}$ is a retract in $B$, in the sense that it has a right inverse $S_{\alpha \beta}$, so $T_{\alpha \beta} S_{\alpha \beta}=1_{H_{\alpha}}$. And $\alpha \leqslant \beta \leqslant$ $\gamma$ implies $S_{\beta \gamma} S_{\alpha \beta}=S_{\alpha \gamma}$. Therefore, each $\lambda_{\delta}$ is a retract.

2.6. Proposition. Let $\left\{H_{\alpha} \stackrel{w_{\alpha \beta}}{\stackrel{1}{\alpha}} H_{\beta}: \alpha, \beta \in D, D\right.$ directed $\}$ be a projective system in $H$. Let $K$ be a Hilbert space and let $\left\{K \stackrel{V_{\delta}}{\longrightarrow} H_{\delta}: \delta \in D\right\}$ be a family of co-isometries which commutes with the system. Then the unique fill-in $\kappa: K \rightarrow$ pro-lim $H_{\delta}$ is surjective. 
PRoof. Let $\left(x_{\delta}\right) \in$ pro-lim $H_{\delta}$. Define $y_{\delta}=V_{\delta}^{*} x_{\delta} \in K$; we show $\left\{y_{\delta}\right\}$ is Cauchy. If $\delta \leqslant \gamma$, then $V_{\gamma} y_{\delta}=V_{\gamma} V_{\delta}^{*} x_{\delta}=V_{\gamma}\left(W_{\delta \gamma} V_{\gamma}\right) * x_{\delta}=V_{\gamma} V_{\gamma}^{*} W_{\delta \gamma}^{*} x_{\delta}=$ $W_{\delta \gamma}^{*} x_{\delta}$. And $\delta \leqslant \gamma$ implies null $V_{\gamma} \subset$ null $V_{\delta}$ and hence (null $\left.V_{\delta}\right)^{\perp} \subset\left(\text { null } V_{\gamma}\right)^{\perp}$. As $y_{\delta} \in \operatorname{ran} V_{\delta}^{*}=\left(\text { null } V_{\delta}\right)^{\perp} \subset\left(\text { null } V_{\gamma}\right)^{\perp}$ we conclude that $y_{\gamma}-y_{\delta} \in\left(\text { null } V_{\gamma}\right)^{\perp}$ and that $V_{\gamma}$ acts isometrically on this vector: $\left\|y_{\gamma}-y_{\delta}\right\|=\left\|V_{\gamma} y_{\gamma}-V_{\gamma} y_{\delta}\right\|=\left\|x_{\gamma}-W_{\delta \gamma}^{*} x_{\delta}\right\|$ and

$$
\begin{aligned}
\left\|x_{\gamma}-W_{\delta \gamma}^{*} x_{\delta}\right\|^{2} & =\left\|x_{\gamma}\right\|^{2}-\left\langle x_{\gamma} \mid W_{\delta \gamma}^{*} x_{\delta}\right\rangle-\left\langle W_{\delta \gamma}^{*} x_{\delta} \mid x_{\gamma}\right\rangle+\left\|W_{\delta \gamma}^{*} x_{\delta}\right\|^{2} \\
& \leqslant\left\|x_{\gamma}\right\|^{2}-\left\langle W_{\delta \gamma} x_{\gamma} \mid x_{\delta}\right\rangle-\left\langle x_{\delta} \mid W_{\delta \gamma} x_{\gamma}\right\rangle+\left\|x_{\delta}\right\|^{2} \\
& =\left\|x_{\gamma}\right\|^{2}-\left\langle x_{\delta} \mid x_{\delta}\right\rangle-\left\langle x_{\delta} \mid x_{\delta}\right\rangle+\left\|x_{\delta}\right\|^{2} \\
& =\left\|x_{\gamma}\right\|^{2}-\left\|x_{\delta}\right\|^{2}
\end{aligned}
$$

which goes to zero as $\gamma, \delta \uparrow$ since $\left\|\left(x_{\delta}\right)\right\|=\lim _{\delta \uparrow}\left\|x_{\delta}\right\|$. Thus $\left\{y_{\delta}\right\}$ is Cauchy in $K$; let $y$ be its limit.

Claim. $V_{\delta} y=x_{\delta}$, all $\delta:$ Fix $\delta \in D$ and $\epsilon>0$; choose $\gamma \geqslant \delta$ such that $\| y-$ $y_{\gamma} \|<\epsilon$. Then

$$
\begin{aligned}
\left\|V_{\delta} y-x_{\delta}\right\| & \leqslant\left\|V_{\delta} y-V_{\delta} y_{\gamma}\right\|+\left\|V_{\delta} y_{\gamma}-x_{\delta}\right\| \\
& \leqslant\left\|y-y_{\gamma}\right\|+\left\|V_{\delta} y_{\gamma}-x_{\delta}\right\|=\left\|y-y_{\gamma}\right\|<\epsilon
\end{aligned}
$$

since $V_{\delta} y_{\gamma}-\underline{x}_{\delta}=w_{\delta \gamma} V_{\gamma} y_{\gamma}-x_{\delta}=w_{\delta \gamma} x_{\gamma}-x_{\delta}=x_{\delta}-x_{\delta}=0$. As $\epsilon$ was arbitrary, $V_{\delta} y=x_{\delta}$. (Q.E.D.)

Finally, $\kappa(y)=\left(V_{\delta}(y)\right)=\left(x_{\delta}\right)$, showing $\kappa$ surjective. Q.E.D. $=\|\kappa(y)\|_{\text {. }}$

Note. The vector $y$ in the proof satisfies $\|y\|=\lim \left\|y_{\delta}\right\|=\lim \left\|x_{\delta}\right\|=\left\|\left(x_{\delta}\right)\right\|$

2.7. COROLLARY. Let $K$ be a Hilbert space and let $\left\{H_{\delta}\right\}$ be a directed set of closed subspaces of $K$, ordered by inclusion, such that under orthogonal projection, $\left\{H_{\alpha} \stackrel{w_{\alpha \beta}}{\longleftarrow} H_{\beta}\right\}$ is a projective system in $H$. If $K=\bigvee_{D} H_{\delta}$ (in the lattice of closed subspaces), then $K \cong$ pro-lim $H_{\delta}$ unitarily.

Proof. Map $K \stackrel{V_{\delta}}{\longrightarrow} H_{\delta}$ by orthogonal projection, which is a co-isometry from $K$ to $H_{\delta}$. The unique fill-in $\kappa: K \rightarrow$ pro-lim $H_{\delta}$ is surjective. And $0=\kappa(y)=$ $\left(V_{\delta}(y)\right) \Rightarrow V_{\delta}(y)=0$, all $\delta \Rightarrow y \in H_{\delta}^{\perp}$, all $\delta \Rightarrow y \in \bigcap H_{\delta}^{\perp}=\left(\bigvee H_{\delta}\right)^{\perp}=K^{\perp}=\{0\}$, so that $\kappa$ is injective. By the preceding note, $\kappa$ must be isometric, hence unitary. Q.E.D.

As before, by taking dual objects and dual morphisms, we obtain results on injective limits in $H$ dual to $2.4,2.5$, and 2.6 , by interchanging the terms isometry and co-isometry. A bounded linear transformation $T: X \rightarrow Y$ between Hilbert spaces is called bounded below if there is a number $M>0$ such that $\|T x\| \geqslant M\|x\|$, all $x \in X$ (in which case $T$ is one-to-one and has closed range). It is known that $T$ 
is surjective $\Leftrightarrow T^{*}$ is bounded below ( $\left.\Leftrightarrow T\right|_{(\text {null } T)^{\perp}}$ is invertible), which fact indicates the correct reformulation of 2.6 .

3. Preservation of projective limits. There is a theorem stating that every compact group is the projective limit (under surjections) of closed subgroups of unitary groups $U(n)(n<\infty)$, and another theorem stating that every locally compact group contains an open subgroup which is the projective limit (under proper surjections) of Lie groups. Consequently, one is interested in whether projective limits are preserved under the application of the (co-variant) functors $G \mapsto L^{2}(G)$, $L^{1}(G), M(G)$ of Theorem 1.7, and a fourth functor, the von Neumann algebra generated by left regular representation of $L^{1}(G)$ on $L^{2}(G)$, which we discuss shortly.

Our general setting will be: a projective system $\left\{G_{\alpha} \stackrel{w_{\alpha \beta}}{\longleftarrow} G_{\beta}: \alpha \leqslant \beta\right.$ in $D, D$ directed $\}$ with limit $\left\{G \stackrel{v_{\delta}}{\longrightarrow} G_{\delta}\right\}$, where the objects are locally compact Hausdorff groups and the morphisms are continuous proper surjective group homomorphisms. (Given $\left\{G_{\alpha} \stackrel{w_{\alpha \beta}}{\longleftarrow} G_{\beta}\right\}$ as described, it is known that the limit of this system, as calculated in the category (topological groups, continuous group homomorphisms), is given by a locally compact Hausdorff group, together with proper surjections.)

Our results on preservation of projective limits will be consequences of the following proposition on injective limits in $B$.

3.1. Proposition. Let $\left\{N_{\alpha} \stackrel{R_{\beta \alpha}}{\longrightarrow} N_{\beta}: \alpha \leqslant \beta\right.$ in $D, D$ directed $\}$ be an injective system in the category $B=$ (Banach spaces, linear contractions) and let $\left\{N_{\delta} \stackrel{R_{\delta}}{\longrightarrow} N\right\}$ be a family in $B$ commuting with this system. If

(i) each $R_{\delta}$ is isometric,

(ii) $\bigcup_{D}$ Range $\left(R_{\delta}\right)$ is dense in $N$, then $\left\{N_{\delta} \stackrel{R_{\delta}}{\longrightarrow} N\right\}$ is the injective limit of $\left\{N_{\alpha} \stackrel{R_{\beta \alpha}}{\longrightarrow} N_{\beta}\right\}$ in $B$.

Proof. Suppose $\left\{N_{\delta} \stackrel{T_{\delta}}{\longrightarrow} A\right\}$ is a family in $B$ commuting with the system. We define a morphism $S: N \rightarrow A$. Given $f \in N$, we may choose a net $\left\{R_{\delta}\left(f_{\delta}\right)\right\}$ from $\bigcup_{D}$ Range $\left(R_{\delta}\right)$ such that $R_{\delta} f_{\delta} \rightarrow f$.

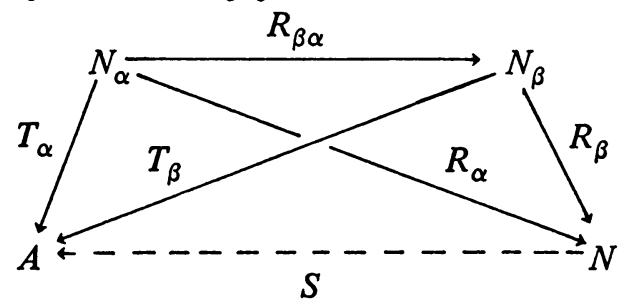


If $\alpha \leqslant \beta$, then $\left\|T_{\alpha} f_{\alpha}-T_{\beta} f_{\beta}\right\|=\left\|T_{\beta} R_{\beta \alpha} f_{\alpha}-T_{\beta} f_{\beta}\right\| \leqslant\left\|R_{\beta \alpha} f_{\alpha}-f_{\beta}\right\|$; since $R_{\beta}$ is isometric, the last term equals $\left\|R_{\beta} R_{\beta \alpha} f_{\alpha}-R_{\beta} f_{\beta}\right\|=\left\|R_{\alpha} f_{\alpha}-R_{\beta} f_{\beta}\right\|$. It follows that $\left\{T_{\delta} f_{\delta}\right\}$ is Cauchy in $A$, let $a \in A$ be its limit and define $S(f)=a$. The usual considerations show that $S$ is well defined, linear, and satisfies $S \circ R_{\delta}=T_{\delta}$, all $\delta$; moreover, $S$ is unique with respect to the last property, by density of $\bigcup_{D}$ Range $\left(R_{\delta}\right)$. Finally,

$$
\|f\|=\lim \left\|R_{\delta} f_{\delta}\right\|=\lim \left\|f_{\delta}\right\| \geqslant \lim \left\|T_{\delta} f_{\delta}\right\|=\|a\|
$$

shows $S$ is contractive. Q.E.D.

Let $*: B \rightarrow B^{o p}$ be the functor which, to a Banach space $A$, assigns the dual space $A^{*}=\{$ continuous linear $p: A \rightarrow C\}$ and which to a morphism $A \stackrel{T}{\rightarrow} B$ in $B$, assigns $B^{*} \stackrel{T^{*}}{\rightarrow} A^{*}$, where $T^{*}(p)(a)=p(T a), p \in B^{*}, a \in A$. (Here $B^{\text {op }}$ is the "opposite category" of $B$; it has the same objects as $B$, and the same morphism as well, except that the direction of arrows is reversed-this is merely a device to incorporate the fact that the functor * reverses the direction of morphisms.) The functor $*$ is left adjoint to itself (we refer to [4] as a reference on adjoint pairs of functors). In our setting, our claim amounts to the following: define $\eta_{E}: E \rightarrow E^{* *}$ by $\eta_{E}(e)(p)=p(e)$ for $e \in E, p \in E^{*}$; then given any B-object $F$ and $B$-morphism $j: E \rightarrow F^{*}$, there is a unique $B$-morphism $J: F \rightarrow E^{*}$ such that $J^{*} \circ \eta_{E}=j$ (namely, $J(f)(e)=j(e)(f)$, for $\left.f \in F, e \in E\right)$.
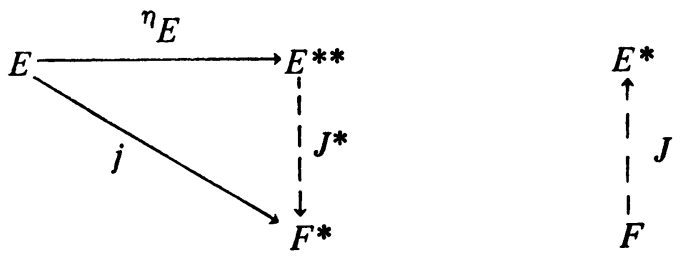

Given an adjoint pair of functors, the right adjoint is continuous (that is, it preserves the limit of any (set-indexed) diagram in its domain category) and the left adjoint is cocontinuous (preserves colimits). As * reverses arrows, it follows that if $\left\{N_{\alpha} \stackrel{R_{\beta \alpha}}{\longrightarrow} N_{\beta}: \alpha \leqslant \beta\right.$ in $D, D$ directed $\}$ is an injective system in $B$ with injective limit $\left\{N_{\delta} \stackrel{R_{\delta}}{\longrightarrow} N\right\}$, then $\left\{N^{*} \stackrel{R_{\delta}^{*}}{\longrightarrow} N_{\delta}^{*}\right\}$ is the projective limit of the projective system $\left\{N_{\alpha}^{*} \stackrel{R_{\beta \alpha}^{*}}{\longleftarrow} N_{\beta}^{*}\right\}$. Likewise, projective limits in $B$ are transformed into injective limits under application of $*$.

We return to our setting of a projective system $\left\{G_{\alpha} \stackrel{w_{\alpha \beta}}{\longleftarrow} G_{\beta}: \alpha \leqslant \beta\right.$ in $D$, $D$ directed $\}$ with limit $\left\{G \stackrel{v_{\delta}}{\longrightarrow} G_{\delta}\right\}$, where the groups are locally compact and the morphisms are continuous proper surjective group homomorphisms.

3.2. Lemma. If $F=\bigcup_{D}\left\{g \circ v_{\delta}: g \in C_{00}\left(G_{\delta}\right), \delta \in D\right\} \subseteq C_{00}(G)$, then $F$ is uniformly dense in $C_{0}(G)$. 
Proof. Given $f \in C_{00}\left(G_{\alpha}\right), f^{\prime} \in C_{00}\left(G_{\beta}\right)$, choose $\gamma \geqslant \alpha, \beta$; then $f \circ v_{\alpha}=$ $f \circ w_{\alpha \gamma} \circ v_{\gamma}, f^{\prime} \circ v_{\beta}=f^{\prime} \circ w_{\beta \gamma} \circ v_{\gamma}$ and we may add, multiply and conjugate in $C_{00}\left(G_{\gamma}\right) ; \bigcap \operatorname{Ker} v_{\delta}=0 \Rightarrow F$ separates; now apply the Stone-Weierstrass theorem. Q.E.D.

By the functorality in Theorem 1.7, we obtain a projective system $\left\{L^{2}\left(G_{\alpha}\right) \stackrel{\Psi_{\alpha \beta}}{\longleftarrow} L^{2}\left(G_{\beta}\right): \alpha \leqslant \beta\right.$ in $\left.D\right\}$ in B (actually in $H$ ), a family $\left\{L^{2}(G) \stackrel{\Psi_{\delta}}{\longrightarrow}\right.$ $\left.L^{2}\left(G_{\delta}\right)\right\}$ commuting with this system, hence a fill-in $U: L^{2}(G) \rightarrow$ pro-lim $L^{2}\left(G_{\delta}\right)$, $U(f)=\left(\Psi_{\delta}(f)\right)_{D}$.

3.3. THEOREM. $L^{2}(G)$ and pro-lim $L^{2}\left(G_{\delta}\right)$ are unitarily isomorphic under $U$.

Proof. We first obtain an injective system $\left\{L^{2}\left(G_{\alpha}\right) \stackrel{R_{\beta \alpha}}{\longrightarrow} L^{2}\left(G_{\beta}\right)\right\}$ and a family $\left\{L^{2}\left(G_{\delta}\right) \stackrel{R_{\delta}}{\longrightarrow} L^{2}(G)\right\}$ commuting with this system, where the $R$-type maps are given by composition with the corresponding group morphism, as in Proposition 1.2. Each $R$-type map is isometric. Lemma 3.2 implies that $\bigcup_{D} \operatorname{Range}\left(R_{\delta}\right)$ is $L^{2}$-dense in $L^{2}(G)$. By $3.1,\left\{L^{2}\left(G_{\delta}\right) \stackrel{R_{\delta}}{\longrightarrow} L^{2}(G)\right\}$ is the injective limit of $\left\{L^{2}\left(G_{\alpha}\right) \stackrel{R_{\beta} \alpha}{\rightarrow} L^{2}\left(G_{\beta}\right)\right\}$. Apply the functor $*$ and identify a Hilbert space $H$ with its dual space $H^{*}$ in the usual way; then the image under $*$ of an $R$-type map is identified with its Hilbert space adjoint, that is, with the corresponding $\Psi$-type map (Theorem 1.3). So $\left\{L^{2}(G) \stackrel{\Psi_{\delta}}{\longrightarrow} L^{2}\left(G_{\delta}\right)\right\}$ is the projective limit of $\left\{L^{2}\left(G_{\alpha}\right) \stackrel{\Psi_{\alpha \beta}}{\longleftarrow} L^{2}\left(G_{\beta}\right)\right\}$. Equivalently, $U$ is an isomorphism. But an isomorphism in $B$ must be isometric (see the paragraph preceding Proposition 2.5). Q.E.D.

The case $M(G)=C_{0}(G) *$ is analogous: in the category $B^{\prime}=($ Banach*. algebras, contractive*-algebra homomorphisms) we obtain a projective system $\left\{M\left(G_{\alpha}\right) \stackrel{\Phi_{\alpha \beta}}{\stackrel{(}{\alpha}} M\left(G_{\beta}\right)\right\}$ and a family $\left\{M(G) \stackrel{\Phi_{\delta}}{\longrightarrow} M\left(G_{\delta}\right)\right\}$ commuting with this system, hence a $B^{\prime}$-morphism $\kappa: M(G) \rightarrow$ pro-lim $M\left(G_{\delta}\right), \kappa(\mu)=\left(\Phi_{\delta}(\mu)\right)_{D}$. The map $G \stackrel{v_{\delta}}{\longrightarrow} G_{\delta}$ being surjective implies that the map $C_{0}\left(G_{\delta}\right) \stackrel{R_{\delta}}{\longrightarrow} C_{0}(G)$, given by composition with $v_{\delta}$, is isometric. As above,

3.4. THEOREM. The map $\kappa: M(G) \rightarrow$ pro-lim $M\left(G_{\delta}\right)$ defined by $\kappa(\mu)=$ $\left(\Phi_{\delta}(\mu)\right)_{D}$ is an isometric*-algebra isomorphism.

We remark that the measure $\mu$ is positive if and only if each $\Phi_{\delta}(\mu)$ is positive.

We next present an example that answers in the negative the question: does the functor $G \rightarrow L^{1}(G)$ preserve projective limits? The answer is not altogether surprising since $L^{1}(G)$ is generally not the dual space of another Banach space.

On the other hand, it is not implausible to conjecture that: if every co- 
ordinate $\mu_{\delta}$ of an element $\bar{\mu}=\left(\mu_{\delta}\right) \in$ pro-lim $M\left(G_{\delta}\right)$ satisfies $\mu_{\delta} \ll \lambda_{G_{\delta}}$, then would not also $\bar{\mu} \ll \lambda_{G}$ ? That this need not be the case is illustrated in our example; the example arises even in the desirable situation that all groups are compact abelian. By definition, the support of a positive measure $\mu$ is the complement of the largest $\mu$-null open set. We need

LEmma. Let $G \stackrel{\varphi}{\rightarrow} H, M(G) \stackrel{\oplus}{\rightarrow} M(H)$ be as in Theorem 1.7, and let $\mu \in$ $M(G)$ be a positive measure. If $A=\operatorname{supp}(\Phi \mu) \subset H$, then $\operatorname{supp}(\mu) \subset \varphi^{-1}(A)$.

PROOF. $\mu(G) \geqslant \mu\left(\varphi^{-1}(A)\right)=\Phi \mu(A)=\Phi \mu(H)=\mu\left(\varphi^{-1}(H)\right)=\mu(G) \Rightarrow \mu(G)$ $=\mu\left(\varphi^{-1}(A)\right) \Rightarrow \operatorname{supp}(\mu) \subset \varphi^{-1}(A)$. Q.E.D.

3.5. EXAMPLE. That $G \mapsto L^{1}(G)$ need not preserve projective limits. Let $T^{1}$ be the circle group, let $T^{n}$, for $n=2,3, \ldots$ (resp., let $T^{\infty}$ ) be the product of $n$ (resp., countably many) copies of $T^{1}$. Map $T^{n} \rightarrow T^{n-1}$ by $\left(x_{1}, \ldots, x_{n}\right)$ $\mapsto\left(x_{1}, \ldots, x_{n-1}\right)$. Then $T^{\infty}$, together with the maps $\left(x_{k}\right)_{k=1}^{\infty} \stackrel{\varphi_{n}}{\longrightarrow}\left(x_{1}, \ldots\right.$, $\left.x_{n}\right)$, is the projective limit of $T^{n-1} \leftarrow T^{n}$. Consequently, Haar measure $\lambda_{T^{\infty}}$ on $T^{\infty}$ is sent to Haar measure $\lambda_{T^{n}}$ on $T^{n}$, which is sent to that of $T^{n-1}$, etc. We obtain a commuting diagram:

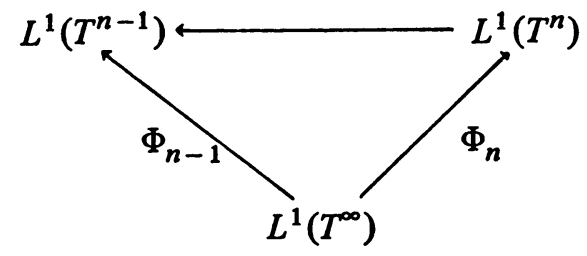

Let $I$ be a closed subset of $T^{1}$ such that $\lambda_{T^{1}}(I)=\alpha^{-1}$ where $\alpha>1$. Let $I^{m}$ denote $I \times \ldots \times I \subset T^{n}$. Set $f_{n}=\alpha^{n} \xi_{I} n \in L^{1}\left(T^{n}\right) ; f_{n}$ is a unit vector, so $\sup \left\|f_{n}\right\|=1<\infty$.

Claim. $L^{1}\left(T^{n}\right) \stackrel{\Phi}{\rightarrow} L^{1}\left(T^{n-1}\right)$ sends $f_{n}$ to $f_{n-1}:$ it suffices to show that $\int \xi_{E}\left(\Phi f_{n}\right) d \lambda_{T^{n-1}}=\int \xi_{E} f_{n-1} d \lambda_{T^{n-1}}$ for Borel $E \subset T^{n-1}$.

$$
\begin{aligned}
& \int_{T^{n-1}} \xi_{E}\left(\Phi f_{n}\right) d \lambda_{T^{n-1}}=\int_{T^{n}}\left(\xi_{E} \circ \varphi\right) f_{n} d \lambda_{T^{n}}=\int_{T^{n}} \xi_{\varphi^{-1}(E)} \alpha^{n} \xi_{I^{n}} d \lambda_{T^{n}} \\
& =\alpha^{n} \int_{T^{n}} \xi_{\left(E \times T^{1}\right) \cap\left(I^{n-1} \times I\right)} d \lambda_{T^{n}}=\alpha^{n} \int_{T^{n}} \xi_{\left(E \cap I^{n-1}\right) \times I} d \lambda_{T^{n}} \\
& =\alpha^{n} \lambda_{T^{n}}\left(\left(E \cap I^{n-1}\right) \times I\right)=\alpha^{n} \lambda_{T^{n-1}}\left(E \cap I^{n-1}\right) \lambda_{T^{1}}(I) \\
& =\alpha^{n-1} \lambda_{T^{n-1}}\left(E \cap I^{n-1}\right)=\int_{T^{n-1} \xi_{E}\left(\alpha^{n-1} \xi_{I^{n-1}}\right) d \lambda_{T^{n-1}}} \\
& =\int_{T^{n-1}} \xi_{E} f_{n-1} d \lambda_{T^{n-1}} \text {. }
\end{aligned}
$$


It follows that $\left(f_{n}\right)_{n=1}^{\infty} \in$ pro-lim $L^{1}\left(T^{n}\right) \subset$ pro-lim $M\left(T^{n}\right) \cong M\left(T^{\infty}\right)$; let $\mu$ be that positive nonzero element of $M\left(T^{\infty}\right)$ satisfying $\Phi_{n} \mu=f_{n}$, all $n$. Then $\operatorname{supp}\left(\Phi_{n} \mu\right)=\operatorname{supp}\left(f_{n}\right)=I^{n} \Rightarrow \operatorname{supp}(\mu) \subset \varphi_{n}^{-1}\left(I^{n}\right) \Rightarrow \lambda_{T^{\infty}}(\operatorname{supp}(\mu)) \leqslant$ $\lambda_{T^{\infty}}\left(\varphi_{n}^{-1}\left(I^{n}\right)\right)=\lambda_{T^{n}}\left(I^{n}\right)=\alpha^{-n}$, which goes to zero as $n$ increases. Thus, $\operatorname{supp}(\mu)$ is a null set with respect to $\lambda_{T^{\infty}}$; consequently, $\mu \notin L^{1}\left(T^{\infty}\right)$, and $L^{1}\left(T^{\infty}\right)$ is a proper subset of pro-lim $L^{1}\left(T^{n}\right)$. Q.E.D.

It can happen that pro-lim $L^{1}\left(G_{\delta}\right)$ is all of pro-lim $M\left(G_{\delta}\right)$. A topological group $G$ is called zero-dimensional if it has a basis of neighborhoods of the identity consisting of open-closed sets. If $G$ is zero-dimensional and compact, then $G$ is the projective limit of finite discrete groups $G_{\delta}$ (and vice versa). Then $G_{\delta}$ discrete implies $L^{1}\left(G_{\delta}\right)=M\left(G_{\delta}\right)$.

We now consider $W^{*}$-algebras. We shall assume familiarity with such algebras, referring the reader to [2] and [5] for details. A $W^{*}$-algebra $N$ is a $C^{*}$. algebra which is the dual space of some other Banach space $N_{*}$, called the predual of $N, N=\left(N_{*}\right)^{*}$. Of necessity, $N$ possesses a (multiplicative) unit element. A *-algebra homomorphism between $W^{*}$-algebras is necessarily contractive, and is called normal if it is continuous with respect to the weak-star topologies on its domain and codomain. We reserve the term von Neumann algebra for an u.w.o.t.closed operator algebra acting on some specified Hilbert space, u.w.o.t. $=$ ultraweak operator topology. Any von Neumann algebra is a $W^{*}$-algebra. If $N$ is a von Neumann algebra, then its predual $N_{*}$ may be identified with the space of u.w.o.t.-continuous linear functionals on $N$ (the functionals carry the uniform norm $\|p\|=\sup \{|p(n)|: n$ is a unit vector in $N\})$, and the ultra-weak operator topology on $N$ is the weak-star topology from $N_{*}$.

Given a locally compact group $G$, let $B\left(L^{2}(G)\right)$ denote the bounded linear operators on the Hilbert space $L^{2}(G)$. As $\|f * w\|_{2} \leqslant\|f\|_{1}\|w\|_{2}$ for $f \in L^{1}(G)$, $w \in L^{2}(G)$, each $f \in L^{1}(G)$ determines an element $\pi(f)$ of $B\left(L^{2}(G)\right)$. The map $f \mapsto \pi(f)$ is a contractive*-algebra homomorphism, called the left regular representation of $L^{1}$ on $L^{2}$. The representation is faithful, so we identify $L^{1}$ with its image.

Notation. $W(G)$ denotes the von Neumann algebra in $B\left(L^{2}(G)\right)$ generated by $L^{1}(G)$ under left regular representation.

Since $L^{1}$ contains an approximate identity $\left\{e_{\alpha}\right\}$ such that $\left\|e_{\alpha} * w-w\right\|_{2}$ $\rightarrow 0$ as $\alpha \uparrow$, all $w \in L^{2}(G)$, it follows that $L^{1}$ acts nondegenerately on $L^{2}(G)$, that the strong operator topology closure of $L^{1}$ contains the identity operator of $L^{2}(G)$, and that $W(G)=$ the closure of $L^{1}$ under any of: the strong (resp., ultrastrong, weak, ultra-weak) operator topologies in $B\left(L^{2}(G)\right)=$ the double commutant of $L^{1}$ in $B\left(L^{2}(G)\right.$ ). (See [2] or [5].) 
Let $\varphi$ : $G \rightarrow H$ be a continuous proper surjective group homomorphism of locally compact groups. The maps $\Phi: L^{1}(G) \rightarrow L^{1}(H), \Psi: L^{2}(G) \rightarrow L^{2}(H)$ of Theorem 1.7 satisfy $(\Phi f) *(\Psi w)=\Psi(f * w), f \in L^{1}, w \in L^{2}$ (Proposition 1.8); we have

LEMmA. null $\Psi$ and (null $\Psi)^{\perp}$ are reducing subspaces of $L^{2}(G)$ for every operator given by an element of $L^{1}(G)$, hence for every operator in $W(G)$.

3.6. Proposition. Considered as a map between subalgebras of $B\left(L^{2}(G)\right)$, $B\left(L^{2}(H)\right)$, resp., $\Phi$ is ultra-weakly continuous. With regard to the respective uniform operator norms, $\|\Phi\| \leqslant 1$.

Proof. A typical ultra-weak neighborhood of 0 in $B\left(L^{2}(H)\right)$ is of the form $U=$ $\left\{T \in B\left(L^{2}(H)\right):\left|\Sigma_{i=1}^{\infty}\left\langle T a_{i} \mid b_{i}\right\rangle\right|<1\right\}$ where $\left\{a_{i}\right\}_{i=1}^{\infty},\left\{b_{i}\right\}_{i=1}^{\infty} \subset L^{2}(H)$ satisfy $\Sigma\left\|a_{i}\right\|^{2}$, $\Sigma\left\|b_{i}\right\|^{2}<\infty$. Since $\Psi$ is co-isometric (see the definition and remarks following Proposition 2.4), we may take $a_{i}=\Psi x_{i}, b_{i}=\Psi y_{i}$, where $x_{i}, y_{i} \in(\text { null } \Psi)^{\perp}$, in which case $\left\|a_{i}\right\|=\left\|\Psi x_{i}\right\|=\left\|x_{i}\right\|,\left\|b_{i}\right\|=\left\|y_{i}\right\|$, hence $\left\{x_{i}\right\}_{i=1}^{\infty},\left\{y_{i}\right\}_{i=1}^{\infty}$ are square-summable in $L^{2}(G)$. Then

$$
\begin{aligned}
\Phi^{-1}(U) & =\left\{f \in L^{1}(G):\left|\sum\left\langle(\Phi f) *\left(\Psi x_{i}\right) \mid \Psi y_{i}\right\rangle\right|<1\right\} \\
& =\left\{f \in L^{1}(G):\left|\sum\left\langle\Psi\left(f * x_{i}\right) \mid \Psi y_{i}\right\rangle\right|<1\right\} \\
& =\left\{f \in L^{1}(G):\left|\sum\left\langle f * x_{i} \mid y_{i}\right\rangle\right|<1\right\}
\end{aligned}
$$

(since $\Psi$ acts isometrically on (null $\Psi)^{\perp}$ ), and the last set is clearly $U^{\prime} \cap L^{1}(G)$ for an ultra-weak neighborhood $U^{\prime}$ of 0 in $B\left(L^{2}(G)\right)$. Now let $\|f\|_{u}$ denote the uniform norm of $f \in L^{1}$ acting on $L^{2}$. Any unit vector in $L^{2}(H)$ can be taken to be of the form $\Psi w$ for a unit vector $w \in L^{2}(G)$. Then $\|(\Phi f) *(\Psi w)\|_{2}=\|\Psi(f * \dot{w})\|_{2} \leqslant\|f * w\|_{2} \leqslant$ $\|f\|_{u}\|w\|_{2} \Rightarrow\|\Phi f\|_{u} \leqslant\|f\|_{u} \Rightarrow\|\Phi\| \leqslant 1$. Q.E.D.

3.7. Proposition. $\Phi$ has a unique extension to an ultra-weakly continuous *-algebra homomorphism $\widetilde{\Phi}: W(G) \rightarrow W(H)$ (that is, to a morphism of $W^{*}$-algebras). Moreover, $\widetilde{\Phi}$ is surjective. The assignment: $G \stackrel{\varphi}{\rightarrow}$ H goes to $W(G) \stackrel{\widetilde{\Phi}}{\rightarrow} W(H)$, is functorial.

Proof. If $T \in W(G)$, then by the Kaplansky Density Theorem there is a net $\left\{T_{\alpha}\right\} \subset L^{1}$ such that $\left\|T_{\alpha}\right\|_{u} \leqslant\|T\|_{u}$, all $\alpha$, and $T_{\alpha} \rightarrow T$ ultra-weakly. Then $\left\{\Phi T_{\alpha}\right\}$ is ultra-weakly Cauchy and norm bounded (by $\|T\|_{u}$ ) in $B\left(L^{2}(H)\right.$ ), hence converges to some $S \in W(H)$. Define $\widetilde{\Phi} T=S$. The first assertion of the proposition now follows (involution and multiplication are, respectively, ultra-weakly and separately ultraweakly continuous in $\left.B\left(L^{2}(G)\right)\right)$. Since $L^{1}(H) \subseteq$ Range $(\widetilde{\Phi}) \subseteq W(H)$ and since Range $(\widetilde{\Phi})$ must be ultra-weakly closed (see the references), $\widetilde{\Phi}$ is surjective. Functoriality of $G \mapsto L^{1}(G)$ yields the last assertion. Q.E.D. 
We note that the intertwining relation $(\widetilde{\Phi} T)(\Psi w)=\Psi(T w), T \in W(G), w \in L^{2}(G)$, continues to hold.

We shall cease to distinguish between $\Phi$ and its extension, and write $\Phi: W(G) \rightarrow$ $W(H)$. There is the following standard

3.8. Fact. If $\Phi: W \rightarrow V$ is a normal *-algebra homomorphism between $W^{*}$-algebras, then there is a central projection $E \in W$ such that $E \cdot W=\{E A: A \in W\}$ is the kernel of $\Phi$, and $\Phi$ is isometric on the complementary ideal $(I-E) \cdot W$.

This fact has the following consequences:

3.8 (1). Let $W_{1}$ and $V_{1}$ denote, respectively, the unit balls of $W$ and $V$. If $\Phi$ is surjective, then $\Phi\left(W_{1}\right)=V_{1}$.

3.8 (2). A $W^{*}$-algebra is called a factor if its center consists of scalar multiples of the identity element. If $W$ is a factor and $\Phi$ is surjective, than $\Phi$ is an isomorphism and $V$ is a factor.

3.8 (3). (See [5] for the definition of types.) Suppose $\Phi$ is surjective. If $W$ is type I finite (respectively, type $\left.\mathrm{I}_{\infty}, \mathrm{II}_{1}, \mathrm{II}_{\infty}, \mathrm{III}\right)$ then $V$ is type I finite (respectively, type $\left.\mathrm{I}_{\infty}, \mathrm{II}_{1}, \mathrm{II}_{\infty}, \mathrm{III}\right)$.

Let $W^{*}$ be the category whose objects are $W^{*}$-algebras and whose morphisms are normal *-algebra homomorphisms (not necessarily unit preserving). This category is known to have products and equalizers, hence is complete. In particular, if $\left\{W_{\alpha} \stackrel{\Phi_{\alpha \beta}}{\stackrel{1}{L}} W_{\beta}\right\}$ is a projective system in $W^{*}$, then this system has a projective limit in $W^{*}$. Moreover, \{ pro-lim $\left.W_{\delta} \stackrel{\lambda_{\delta}}{\longrightarrow} W_{\delta}\right\}$ as defined in paragraph 2.2 serves as the limiton this point we must exert a little care. Clearly pro-lim $W_{\delta}$ is a $C^{*}$-algebra. Given a morphism $W \stackrel{\Phi}{\rightarrow} V$ in $W^{*}$, we obtain a morphism $V_{*} \stackrel{R}{\rightarrow} W_{*}$ in $B$ between the preduals $\left(R(\sigma)=\sigma \circ \Phi, \sigma \in V_{*}\right)$ whose image under the functor $*$ is $W \stackrel{\Phi}{\rightarrow} V$. The injective system $\left\{\left(W_{\alpha}\right)_{*} \stackrel{R_{\beta \alpha}}{\longrightarrow}\left(W_{\beta}\right)_{*}\right\}$ in $B$ must have an injective limit. Apply $*$ to conclude that pro-lim $W_{\delta}$ is a dual space. (We omit the demonstration that the limit maps $\lambda_{\delta}$ are normal.)

We return to our setting: $\left\{G \stackrel{v_{\delta}}{\longrightarrow} G_{\delta}\right\}$ is the projective limit of the projective system $\left\{G_{\alpha} \stackrel{w_{\alpha \beta}}{\stackrel{ }{\alpha}} G_{\beta}: \alpha, \beta \in D\right\}$, all groups being locally compact, all morphisms being continuous proper surjective group homomorphisms. We have commuting diagrams:
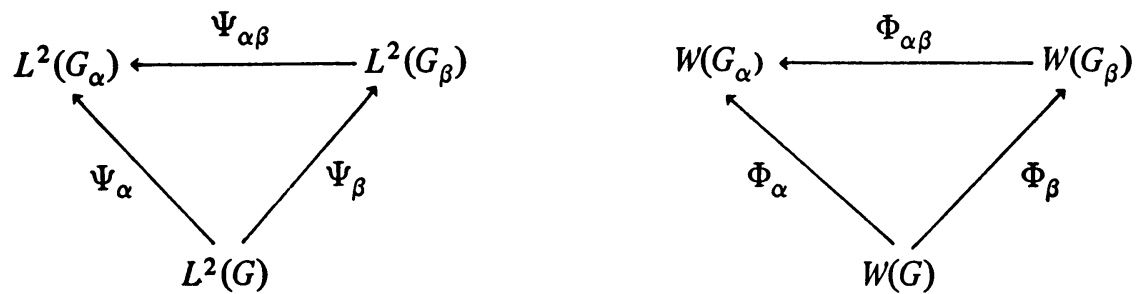

We wish to assert that $\left\{W(G) \stackrel{\Phi_{\delta}}{\longrightarrow} W\left(G_{\delta}\right)\right\}$ is the projective limit of $\left\{W\left(G_{\alpha}\right) \stackrel{\Phi_{\alpha \beta}}{\longleftarrow} W\left(G_{\beta}\right)\right\}$. 
We can say a little more. Each element $\left(T_{\delta}\right) \in$ pro-lim $W\left(G_{\delta}\right)$ determines a bounded linear operator on the Hilbert space pro-lim $L^{2}\left(G_{\delta}\right)$ under $\left(T_{\delta}\right)\left(x_{\delta}\right)=\left(T_{\delta} x_{\delta}\right)$, for $\alpha \leqslant$ $\beta \Rightarrow \Psi_{\alpha \beta}\left(T_{\beta} x_{\beta}\right)=\left(\Phi_{\alpha \beta} T_{\beta}\right)\left(\Psi_{\alpha \beta} x_{\beta}\right)=T_{\alpha} x_{\alpha}$. Let $U: L^{2}(G) \rightarrow$ pro-lim $L^{2}\left(G_{\delta}\right)$ be the unitary map of Theorem 3.3,U(w) $=\left(\Psi_{\delta} w\right), w \in L^{2}(G)$. Let

$$
\kappa: W(G) \rightarrow \text { pro-lim } W\left(G_{\delta}\right)
$$

be the fill-in, $\kappa(T)=\left(\Phi_{\delta} T\right), T \in W(G)$.

3.9. THEOREM. $\left\{W(G) \stackrel{\Phi_{\delta}}{\longrightarrow} W\left(G_{\delta}\right)\right\}$ is the projective limit of $\left\{W\left(G_{\alpha}\right) \stackrel{\Phi_{\alpha \beta}}{\stackrel{1}{\leftrightarrows}} W\left(G_{\beta}\right)\right\}$, and the fill-in $\mathrm{K}$ is a spatial isomorphism under the unitary $U$.

PRoof. As above, in $B$ we have an injective system $\left\{W\left(G_{\alpha}\right)_{*} \stackrel{R_{\beta \alpha}}{\longrightarrow} W\left(G_{\beta}\right)_{*}\right\}$ and a family $\left\{W\left(G_{\delta}\right)_{*} \stackrel{R_{\delta}}{\longrightarrow} W(G)_{*}\right\}$ commuting with this system, where each $R$-type map is given by composition with corresponding $\Phi$-type map. Assertion 3.8 (1) implies that each $R_{\delta}$ is isometric.

Claim. U Range $\left(R_{\delta}\right)$ is norm dense in $W(G)_{*}$.

Proof. $\alpha \leqslant \beta \Rightarrow$ null $\Psi_{\beta} \subseteq$ null $\Psi_{\alpha}$. From Theorem 3.3 it follows that $\bigcup\left(\text { null } \Psi_{\delta}\right)^{\perp}$ is an $L^{2}$-dense linear subspace of $L^{2}(G)$. Let $x \in\left(\text { null } \Psi_{\delta}\right)^{\perp}$. Let $p$ be the positive, weakly continuous linear functional in $W(G)_{*}$ given by $p(T)=\langle T x \mid x\rangle$, for $T \in W(G) ;\|p\|=\|x\|^{2}$. Let $q \in W\left(G_{\delta}\right)_{*}$ be defined by $q(S)=\left\langle S\left(\Psi_{\delta} x\right) \mid \Psi_{\delta} x\right\rangle$, for $S \in W\left(G_{\delta}\right)$. Then $\left(R_{\delta} q\right)(T)=\left(q \circ \Phi_{\delta}\right)(T)=\left\langle\left(\Phi_{\delta} T\right)\left(\Psi_{\delta} x\right) \mid \Psi_{\delta} x\right\rangle=$ $\left\langle\Psi_{\delta}(T x) \mid \Psi_{\delta} x\right\rangle=\langle T x \mid x\rangle$ (since $\Psi_{\delta}$ is isometric on (null $\left.\Psi_{\delta}\right)^{\perp}$ ). Thus $R_{\delta}(q)=p$. It follows that the norm closure of $\bigcup$ Range $\left(R_{\delta}\right)$ in $W(G)_{*}$ contains every weakly continuous linear function in $W(G)_{*}$, hence this closure must equal $W(G)_{*}$. (End of proof of claim.) As in the demonstration of Theorems 3.3 and 3.4, we conclude that $\left\{W(G) \stackrel{\Phi_{\delta}}{\longrightarrow} W\left(G_{\delta}\right)\right\}$ is the projective limit of $\left\{W\left(G_{\alpha}\right) \stackrel{\Phi_{\alpha \beta}}{\longleftarrow} W\left(G_{\beta}\right)\right\}$. Equivalently, $\kappa$ is an isomorphism. That $\kappa$ is spatial via $U$ is now clear. Q.E.D.

\subsection{Proposition. Let $\left\{W_{\alpha} \stackrel{\Phi_{\alpha \beta}}{\longleftarrow} W_{\beta}\right\}$ be a projective system in $\omega^{*}$ with} limit $\left\{\right.$ pro-lim $\left.w_{\delta} \stackrel{\lambda_{\delta}}{\longrightarrow} w_{\delta}\right\}$. If each $\Phi_{\alpha \beta}$ is surjective, then each $\lambda_{\delta}$ is surjective.

Proof. Let $W \stackrel{\Phi}{\rightarrow} V \stackrel{\Gamma}{\rightarrow} X$ be in $W^{*}$, with $\Phi$ and $\Gamma$ assumed surjective. We use Fact 3.8. Let $E \in W$ be the central projection for which $(I-E) \cdot W=\operatorname{Ker} \Phi$. Let $\Phi^{\sim}$ denote the inverse of $\left.\Phi\right|_{E} \cdot W$, so that $\Phi \Phi^{\sim}$ is the identity function of $V$. Let $F \in V$ be the central projection for which $(I-F) \cdot V=\operatorname{Ker} \Gamma$. Let $E_{0} \in W$ be the central projection satisfying $E_{0} \leqslant E$ and $\Phi\left(E_{0}\right)=F$. Clearly then $\left(I-E_{0}\right) \cdot W$ $=\operatorname{Ker}(\Gamma \Phi)$. Thus $(\Gamma \Phi)^{\sim}=\Phi^{\sim} \Gamma^{\sim}$. By the remark after Proposition 2.5, each $\lambda_{\delta}$ is a retract, hence surjective. Q.E.D. 
3.11. THEOREM. Let $\left\{W_{\alpha} \stackrel{\Phi_{\alpha \beta}}{\longleftarrow} W_{\beta}\right\}$ be a projective system in $W^{*}$ with limit \{pro-lim $\left.W_{\delta} \stackrel{\lambda_{\delta}}{\longrightarrow} W_{\delta}\right\}$. Suppose each $\Phi_{\alpha \beta}$ is surjective.

(a) pro-lim $W_{\delta}$ is type $\mathrm{I}$ finite (resp., type $\mathrm{I}_{\infty}, \mathrm{II}_{1}, \mathrm{II}_{\infty}, \mathrm{III}$ ) if and only if each $W_{\delta}$ is type I finite (resp., type $\left.\mathrm{I}_{\infty}, \mathrm{II}_{1}, \mathrm{II}_{\infty}, \mathrm{III}\right)$.

(b) pro-lim $W_{\delta}$ is a factor if and only if each $W_{\delta}$ is a factor.

Proof. The forward implications in (a) and (b) are immediate from 3.8(2), 3.8(3), and 3.10.

(a) Suppose each $W_{\delta}$ is type I finite (the other cases are analogous). By way of contradiction, suppose pro-lim $W_{\delta}$ has a summand of, say, type $\mathrm{I}_{\infty}$ given by the (nonzero) central projection $E$. Since $\bigcap \operatorname{Ker} \lambda_{\delta}=\{0\}$, we may choose $\alpha$ such that $\lambda_{\alpha}(E) \neq 0$. Surjectivity of $\lambda_{\alpha}$ implies that $W_{\alpha}$ has a summand of type $I_{\infty}$, contradiction.

(b) Each $W_{\delta}$ a factor implies that each $\Phi_{\alpha \beta}$ is an isomorphism; then a straightforward categorical argument shows that each limit map $\lambda_{\delta}$ is an isomorphism. Q.E.D.

For a locally compact group $G$, it is known that $W(G)$ decomposes into a summand of type $\mathrm{I}$ and a summand of type $\mathrm{II}_{1}$. In our setting we have

3.12. COROLLARY $W(G)$ is type I (resp., type $\mathrm{II}_{1}$ ) if and only if each $W\left(G_{\delta}\right)$ is type I (resp., type $\left.\mathrm{II}_{1}\right)$.

CONCLUDING REMARKS . (1) Corollary 3.12 recalls the results of Kaniuth [6d], [6c] (see also Smith [6e]) who completely characterized SIN-groups $G$ (SIN = "small invariant neighborhoods") for which $W(G)$ is purely of type I or of type $\mathrm{II}_{1}$. SIN-groups have projective limit characterizations (see [6b]); it remains a future project of ours to investigate to what extent Kaniuth's results may follow from projective limit considerations.

(2) The proof of our Proposition 3.10 gives a technique by which an injective system in $W^{*}$ can be constructed from a given projective system in $W^{*}$. Takeda [6f] and Glimm [6a] have investigated injective limits of $C^{*}$-algebras; both of those authors required their morphisms to be unit-preserving, which condition would not generally hold if the cited technique is used.

(3) Excepting 3.1, 3.10, 3.11, and 3.12, the results in this paper appeared in the author's doctoral dissertation. There the proofs of $3.3,3.4$, and 3.9 were more analytical and did not use the working Proposition 3.1. The author again wishes to thank Professor Karl H. Hofmann of Tulane University, New Orleans, under whose direction the author's dissertation was written.

\section{BIBLIOGRAPHY}

1. N. Bourbaki, Éléments de mathématique. Fasc. II. Livre III: Topologie générale. Chap. 1: Structures topologiques; Chap. 2 : Structures uniformes, 4 ième éd., Actualités Sci. Indust., no. 1142, Hermann, Paris, 1965. MR 39 \#6237.

2. J. Dixmier, Les algébres d'opérateurs dans l'espace hilbertien (algèbres de von Neumann), 2ième éd., Cahiers Scientifique, fasc. 25, Gauthier-Villars, Paris, 1969. 
3. E. Hewitt and K. A. Ross, Abstract harmonic analysis. Vol. I: Structure of topological groups. Integration theory, group representations, Die Grundlehren der math. Wissenschaften, Band 115, Academic Press, New York; Springer-Verlag, Berlin, 1963. MR 28 \#1 58.

4. S. Mac Lane, Categories for the working mathematician, Springer-Verlag, Berlin and New York, 1971.

5. S. Sakai, $C^{*}$-algebras and $W^{*}$-algebras, Springer-Verlag, Berlin and New York, 1971.

6a. J. G. Glimm, On a certain class of operator algebras, Trans. Amer. Math. Soc. 95 (1960), 318-340. MR 22\#2915.

6b. S. Grosser and M. Moskowitz, Compactness conditions in topological groups, J.

Reine Angew. Math. 246 (1971), 1-40. MR 44 \#1766.

6c. E. Kaniuth, Der Typ der regulären Darstellung diskreter Gruppen, Math. Ann. 182 (1969), 334-339. MR 41 \#5516.

6d. - Die Struktur der regulären Darstellung localkompakter Gruppen mit invarianter Umgebungsbasis der Eins, Math. Ann. 194 (1971), 225-248. MR 45 \#2082.

6e. M. Smith, Regular representations of discrete groups, J. Functional Analysis 11 (1972), 401-406.

6f. Z. Takeda, Inductive limit and infinite direct product of operator algebras, Tôhoku Math. J. (2) 7 (1955), 67-86. MR 17, 648.

DEPARTMENT OF MATHEMATICS, UNIVERSITY OF KANSAS, LAWRENCE, KANSAS 66044

Current address: Department of Mathematics, University of North Carolina at Charlotte, UNCC Statior., Charlotte, North Carolina 28213 